See discussions, stats, and author profiles for this publication at: https://www.researchgate.net/publication/308144349

\title{
Inferior frontal gyrus links visual and motor cortices during a visuomotor precision grip force task
}

Article in Brain Research · September 2016

DOI: 10.1016/j.brainres.2016.09.011

CITATIONS

18

6 authors, including:

Christos Papadelis

Cook Children's Health Care System

123 PUBLICATIONS 1,837 CITATIONS

SEE PROFILE

Giandomenico Nollo

Università degli Studi di Trento

251 PUBLICATIONS 3,598 CITATIONS

SEE PROFILE

Some of the authors of this publication are also working on these related projects:

Emotional Processing in Adults and Children View project

Project IORT filter View project

\section{READS}

278

Carola Arfeller

University of Tuebingen

9 PUBLICATIONS 236 CITATIONS

SEE PROFILE

Luigi Cattaneo

Università degli Studi di Trento

117 PUBLICATIONS 4,309 CITATIONS

SEE PROFILE 


\title{
Inferior frontal gyrus links visual and motor cortices during a visuomotor precision grip force task
}

\author{
Christos Papadelis $^{\mathrm{a}, *}$, Carola Arfeller ${ }^{\mathrm{b}}$, Silvia Erla ${ }^{\mathrm{a}, \mathrm{c}}$, Giandomenico Nollo ${ }^{\mathrm{c}}$, Luigi Cattaneo ${ }^{\mathrm{a}}$, Christoph Braun ${ }^{\text {a, b, d }}$ \\ a Laboratory of Functional Neuroimaging, Center for Mind/Brain Sciences (CIMeC), University of Trento, Via delle Regole 101, 38123 Mattarello, Italy \\ ${ }^{\mathrm{b}}$ MEG Center, University of Tübingen, Otfried-Müller-Str 47, 72076 Tübingen, Germany \\ ${ }^{c}$ Biophysics and Biosignals Lab, Department of Physics \& Biotech, University of Trento, Via delle Regole 101, 38123 Mattarello, Italy \\ ${ }^{\mathrm{d}}$ Werner Reichardt Centre for Integrative Neuroscience (CIN) at the University of Tübingen, Otfried-Müller-Str. 25, 72076 Tübingen, Germany
}

\section{A R T I C L E I N F O}

\section{Article history:}

Received 22 April 2016

Received in revised form 6 September 2016

Accepted 7 September 2016

Available online $\mathrm{xxx}$

Keywords:

Visuomotor integration

Coherence

Functional connectivity

Inferior frontal gyrus

Magnetoencephalography

\begin{abstract}
A B S T R A C T
Coordination between vision and action relies on a fronto-parietal network that receives visual and proprioceptive sensory input in order to compute motor control signals. Here, we investigated with magnetoencephalography (MEG) which cortical areas are functionally coupled on the basis of synchronization during visuomotor integration. MEG signals were recorded from twelve healthy adults while performing a unimanual visuomotor (VM) task and control conditions. The VM task required the integration of pinch motor commands with visual sensory feedback. By using a beamformer, we localized the neural activity in the frequency range of $1-30 \mathrm{~Hz}$ during the VM compared to rest. Virtual sensors were estimated at the active locations. A multivariate autoregressive model was used to estimate the power and coherence of estimated activity at the virtual sensors. Event-related desynchronisation (ERD) during VM was observed in early visual areas, the rostral part of the left inferior frontal gyrus (IFG), the right IFG, the superior parietal lobules, and the left hand motor cortex (M1). Functional coupling in the alpha frequency band bridged the regional activities observed in motor and visual cortices (the start and the end points in the visuomotor loop) through the left or right IFG. Coherence between the left IFG and left M1 correlated inversely with the task performance. Our results indicate that an occipital-prefrontal-motor functional network facilitates the modulation of instructed motor responses to visual cues. This network may supplement the mechanism for guiding actions that is fully incorporated into the dorsal visual stream.
\end{abstract}

(C) 2016 Published by Elsevier Ltd

\section{Introduction}

The ability to continuously adapt motor output to sensory feedback is crucial to everyday functioning and is utilized in tasks ranging from simple to more complex. The continuous adjustment of a force to a visual feedback requires the integration of sensory input and motor output, and it involves the continuous integration of activity from different frontal, parietal, and sensorimotor brain regions in an extended brain network (Floyer-Lea and Matthews, 2004; Vaillancourt et al., 2006).

\footnotetext{
Abbreviations: AIP, anterior intraparietal area; DLPFC, dorsolateral prefrontal cortex; fMRI, functional magnetic resonance imaging; IFG, inferior frontal gyrus; IFS, inferior frontal sulcus; IOFF, inferior occipito-frontal fascicle; IPS, intraparietal sulcus; M, pure motor; M1, hand motor cortex; MEG, magnetoencephalography; MVAR, multivariate autoregressive; PCM, probabilistic cytoarchitectonic maps; PET, positron emission tomography; PMV, ventral premotor cortex; ROI, regions of interest; SAM, Synthetic Aperture Magnetometry; SII, secondary somatosensory cortex; SnPM, Statistical nonParametric Mapping; SLF, superior longitudinal fasciculus; SPL, superior parietal lobe; TMS, transcranial magnetic stimulation; V, pure visual; VM, visuomotor task

* Correspondence to: Fetal-Neonatal Neuroimaging \& Developmental Science Center, Boston Children's Hospital, Harvard Medical School, 9 Hope Avenue, Waltham, MA 02453, USA.
}

Email address: christos.papadelis@childrens.harvard.edu (C. Papadelis)
Previous attempts to understand the electrophysiology of the brain network underlying visuomotor integration have shown a decrease in oscillatory activity, in particular in the alpha and lower beta $(8-21 \mathrm{~Hz})$ frequency bands (Classen et al., 1998; Rearick et al., 2001; Kranczioch et al., 2008; Rilk et al., 2011). These power changes were localized by electroencephalography (EEG) in central, parietal, and occipital brain areas (Classen et al., 1998; Rilk et al., 2011). The communication among these areas was assumed to be implemented through a long-range synchronization mechanism that involves the coherence of neuronal activity across the involved distant brain regions in the alpha (Classen et al., 1998; Chen et al., 2003; Rilk et al., 2011; Mylonas et al., In press), beta and gamma frequency-bands (Aoki et al., 1999; 2001; Babiloni et al., 2006; Baker et al., 1999; Lee, 2003; Ohara et al., 2000; Mylonas et al., In press).

Despite a fair understanding of the neural mechanisms underlying visual and motor systems, the neurophysiological mechanisms which are involved in sensory-motor integration are still unclear. Sensory-motor integration refers to the ability to coherently organize bodily sensations and transfer them into motor commands. The goal of this study is to investigate the neurophysiological patterns of local, intraregional, and interregional oscillatory activity related to sensory-motor integration in a visuomotor precision pinch force task, and to correlate this activity with behavioral performance. The study focuses on the precision pinch because it is a physiologically particularly important motor action. It has been developed in primates for the manipulation of small objects with the tips of the thumb and fingers, and 
requires independent finger movements that involve fine control of the directions and magnitudes of fingertip forces (Flanagan et al., 1999; Johansson et al., 1996). These precise movements are often guided by somatosensory, proprioceptive, and visual feedback. We hypothesize that sensorimotor integration will be executed through a complex functional network that involves primary sensory and motor areas as well as secondary integration areas in the frontal and parietal cortex. We assume that the spatially distributed information between these areas will be integrated into coherent representation states through synchronized rhythmic neural firing, i.e. the integration of distributed information into a unified representation (Gray et al., 1989; Singer, 1999; von der Malsburg, 1999).

To test our hypothesis, we recorded magnetoencephalography (MEG) signals from healthy adult individuals while performing a visuomotor precision pinch force task (VM) as well as during two control conditions involving either pure motor $(\mathrm{M})$ or pure visual (V) tasks. During the VM task, adult participants had to continuously adjust the isometric pinch force with which a manipulandum was held between their right index finger and their thumb (Fig. 1). The requested and the generated force were presented as a visual signal to the participants in order to provide continuous feedback about the accuracy of the generated force. Performance during the VM task was quantified as the continuous tracking error. MEG data analysis was performed on the ongoing cortical activities. By using a beamformer source-localization technique, we estimated the task-related brain oscillatory power changes between 1 and $30 \mathrm{~Hz}$ during VM compared to rest. The power changes were described as event-related synchronization (ERS) or event-related desynchronization (ERD) depending on whether oscillatory power was increased or decreased during the task relative to rest (Aranibar and Pfurtscheller, 1978). Virtual sensors were then estimated at the active locations. A multivariate autoregressive model was used to estimate the power and coherence of estimated activity at the virtual sensors for all three tasks (VM, M, and V). The correlation between the VM task performance and the coherence of brain activity time-courses was also estimated.

\section{Results}

\subsection{Brain source activity estimated by source-reconstruction technique}

Fig. 2 shows an ERD during the VM compared to rest as revealed by group volumetric statistical analysis $(p<0.05)$ with peak locations summarized in Table 1. No significant ERS were observed. For the sake of clarity, we will refer to the brain regions with significant ERD as "active sources" since ERD is generally related to increased neural activation (Pfurtscheller and Lopes da Silva, 1999; Singh et al., 2002; 2003; Neuper et al., 2006).

Active sources were observed (see Table 1): (i) around the rostral part of the left inferior frontal sulcus (this spot was well rostral to the probabilistic location of cytoarchitectonic area BA45 and likely corresponded to BA46 and/or BA10 and will be referred to as rostral IFG see Fig. 2, upper left panel), (ii) in the middle portion of the right IFG falling within the rostral boundaries of BA45 (see Fig. 2, upper left panel), (iii) around the parietal opercular region, most likely representing activation within the secondary somatosensory cortex (SII), corresponding to cytoarchitectonic OP4 subdivision, (iv) in the occipital convexity bilaterally, corresponding to BA18 cytoarchitectonic subdivision, and representing activation within early visual areas (see Fig. 2, upper right panel), (v) in the left precentral sulcus corresponded to the primary motor cortex (M1) within the BA4 (see Fig. 2, lower left panel), and (vi) around the right superior parietal lobe (SPL) that corresponded to cytoarchitectonic area BA7 (see Fig. 2, lower right panel)

\subsection{Spectral changes}

Task-related power changes indicate intraregional oscillatory activity of localized neuronal assemblies. ERD between the VM and the rest was observed for all the virtual sensors $(p<0.05)$ for both alpha and beta frequency bands (see Figs. 3 and 4). Fig. 3 shows the spectrum and the corresponding power changes in the alpha and beta

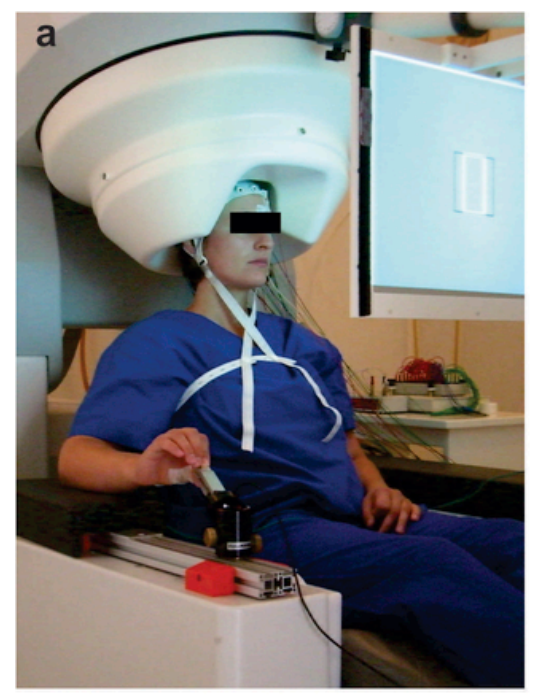

b

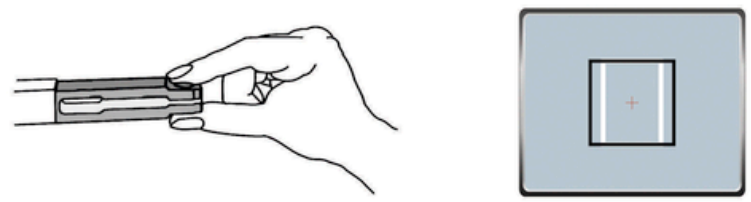

C
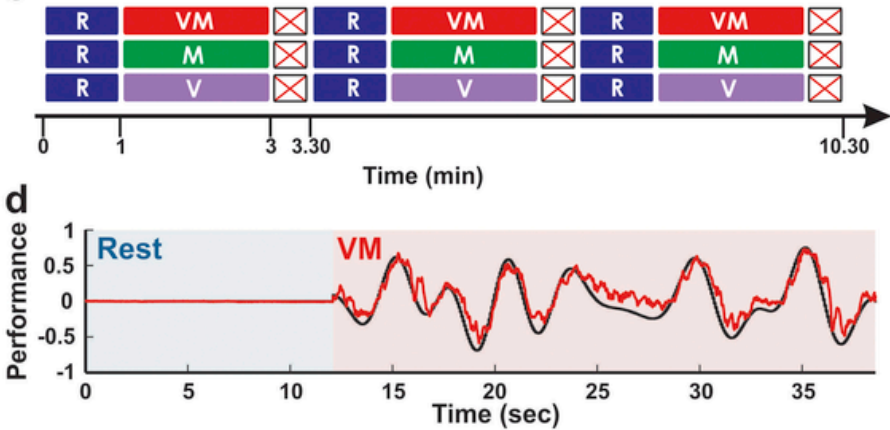

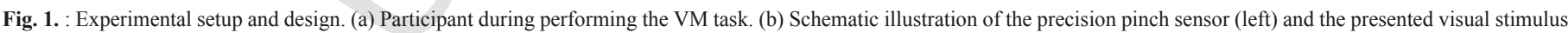

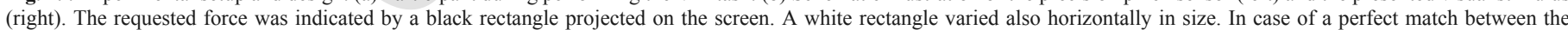

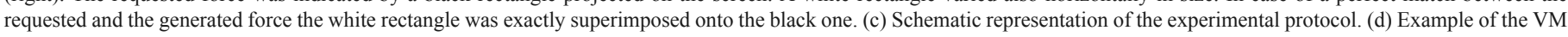

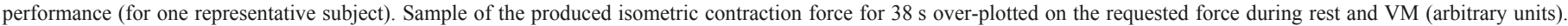
The correlation between the two signals was 0.91 for this subject. 

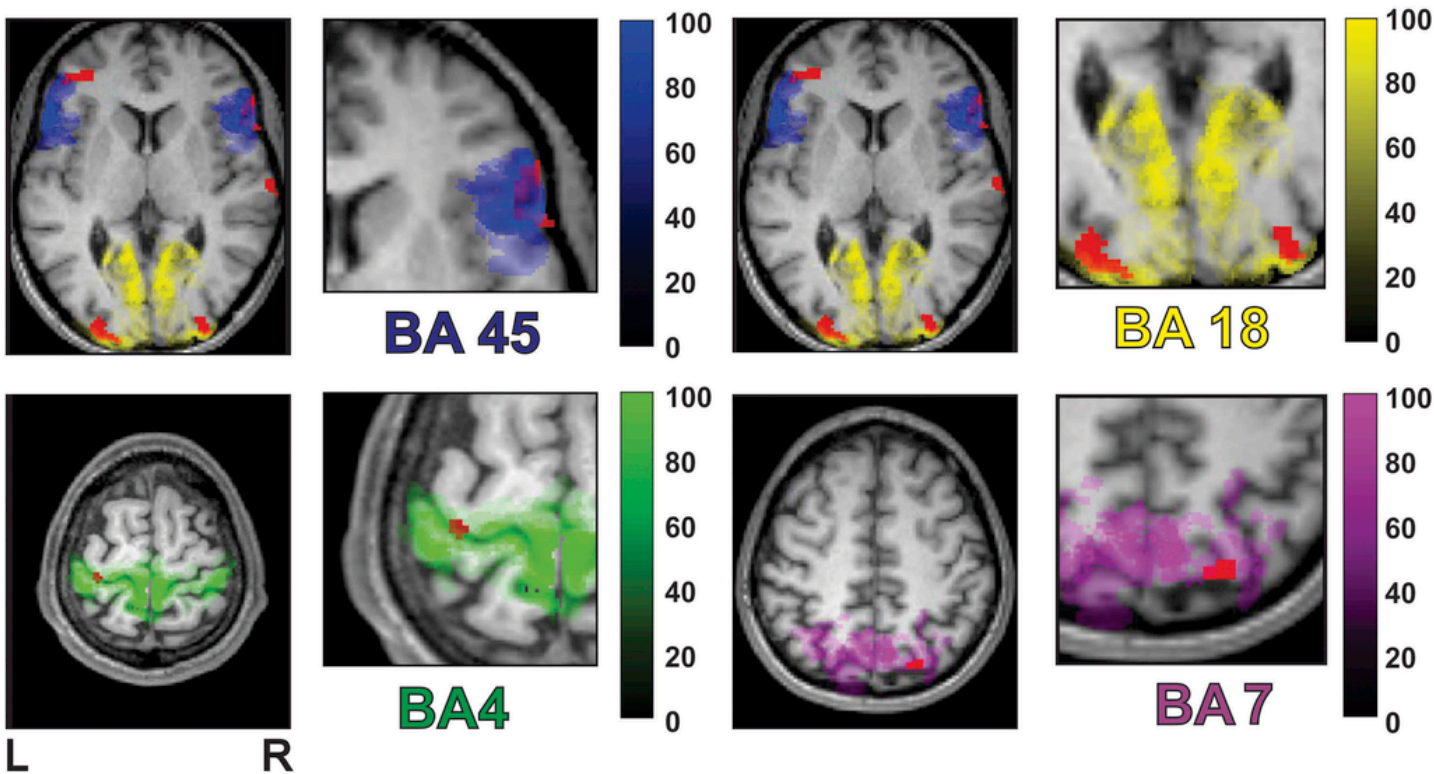

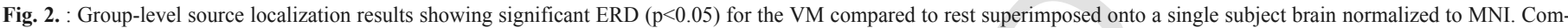

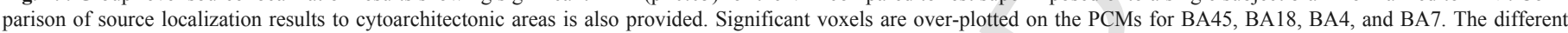
colored scales show the cytoarchitectonic probability for each map (first row: blue for BA-45 and yellow for BA18, second row: green for BA-4 and magenta for BA7).

Table 1

Active sources during VM vs. rest.

\begin{tabular}{llllll}
\hline Anatomic Location & \multicolumn{2}{l}{ MNI Coordinates } & Area & Prob. (\%) \\
\hline & $\mathrm{X}$ & $\mathrm{Y}$ & $\mathrm{Z}$ & & \\
\hline L Inferior occipital gyrus & -24 & -98 & -6 & BA18 & 40 \\
R Inferior occipital gyrus & 31 & -98 & -11 & BA18 & 50 \\
L Rostral IFG & -40 & 44 & 10 & - & - \\
R Middle IFG & 56 & 19 & 10 & BA45 & 80 \\
L Precentral gyrus & -50 & -11 & 46 & BA4a & 50 \\
L Superior Parietal Lobe & -13 & -73 & 56 & SPL-7A & 50 \\
R Superior Parietal Lobe & 24 & -68 & 57 & SPL-7A & 50 \\
R Postcentral gyrus & 62 & -7 & 8 & OP4 & 80 \\
\hline
\end{tabular}

Active sources (anatomical location) during VM compared to rest. Anatomical locations were related to their MNI coordinates and to cyto-architectonic areas with the corresponding probability (Pr.). L: left, R: right.

bands at sensors placed in the left visual area (BA18), the left IFG, and the left M1 (BA4). Fig. 4 shows the power changes in the same bands for the rest of the sensors.

Compared to rest, ERD was seen in the alpha band $(\mathrm{p}<0.05)$ during $\mathrm{V}$ in the left visual (BA18) (see Fig. 3), and the right visual (BA18) and right SII (see Fig. 4). During M, significant alpha power decrease $(p<0.05)$ was observed for the left visual area (BA18), the left IFG, and the left M1 (see Fig. 3), and the right and the left SPL (see Fig. 4).

Compared to rest, ERD in the beta band was decreased $(\mathrm{p}<0.05)$ during V in the left M1 (see Fig. 3), and the right and left SPL (see Fig. 4). Significant power decrease compared to rest $(\mathrm{p}<0.05)$ was observed during M vs. rest only for the sensor at the left M1 (see Fig. 3). For the remaining active sources no significant differences were found between $\mathrm{V}$ or $\mathrm{M}$ and rest.

\subsection{Coherence changes}

Task-related coherence changes indicate intra-regional functional coupling of oscillatory neuronal activity. Coherence analysis was performed for all pairs of virtual sensors. Coherence changes which were attributed specifically to the VM task were considered as those that were significant only for $\mathrm{VM}$ and not for the $\mathrm{V}$ or $\mathrm{M}$ conditions in a specific virtual sensor and involved regions that bridged the regional activity between the motor and visual areas (the start and the end points in the visuomotor loop). Coherence results for three representative pairs of brain source activity (between the left IFG and left visual, the left IFG and left M1, and the left visual and right IFG) are illustrated in Fig. 5 for all experimental conditions. Functional coupling bridged the regional activity between the primary motor and the early visual cortices (the start and the end points in the visuomotor loop) exclusively through the left or right IFG in the alpha band.

Significant results were obtained only for the alpha band. Significant increase $(p<0.05)$ of coherence in the alpha band was observed during VM compared to rest between the left IFG and the source activity time-courses at the left M1, the left and right visual areas, and the left SPL (see Fig. 6 - left upper panel). For the remaining pairs involving the left IFG no significant increase of coherence was found between VM and rest. Coherence in the pair left IFG-right SPL was marginally significant $(p=0.0522)$. Significant increase $(p<0.05)$ of coherence in the alpha band was observed between the right IFG and the source activity time-courses at the left visual and the left M1 (see Fig. 6 - right upper panel). During V, no significant differences between baseline and active period were observed. During condition $\mathrm{M}$, significant coherence was observed $(\mathrm{p}<0.05)$ between the left IFG and the right SPL (see Fig. 6 - lower row).

\subsection{Task performance and its correlation with coherence}

Subjects showed an overall high performance in behavioral measures (correlation between the requested and the performed pinch force $>0.5$ in 9 subjects) during the VM task (mean correlation \pm standard deviation across subjects: $0.74 \pm 0.13, \mathrm{p}<0.01$ ) (see Fig. $1 \mathrm{~d}$ for the performance of one representative subject). Significant negative correlation was observed $(\mathrm{r}=-0.639, \mathrm{p}<0.05)$ between the task performance (correlation between the requested and the performed pinch force) and the coherence of brain activity time-courses for the pair left IFG-left M1 during VM for the alpha band (Fig. 7), but not dur- 


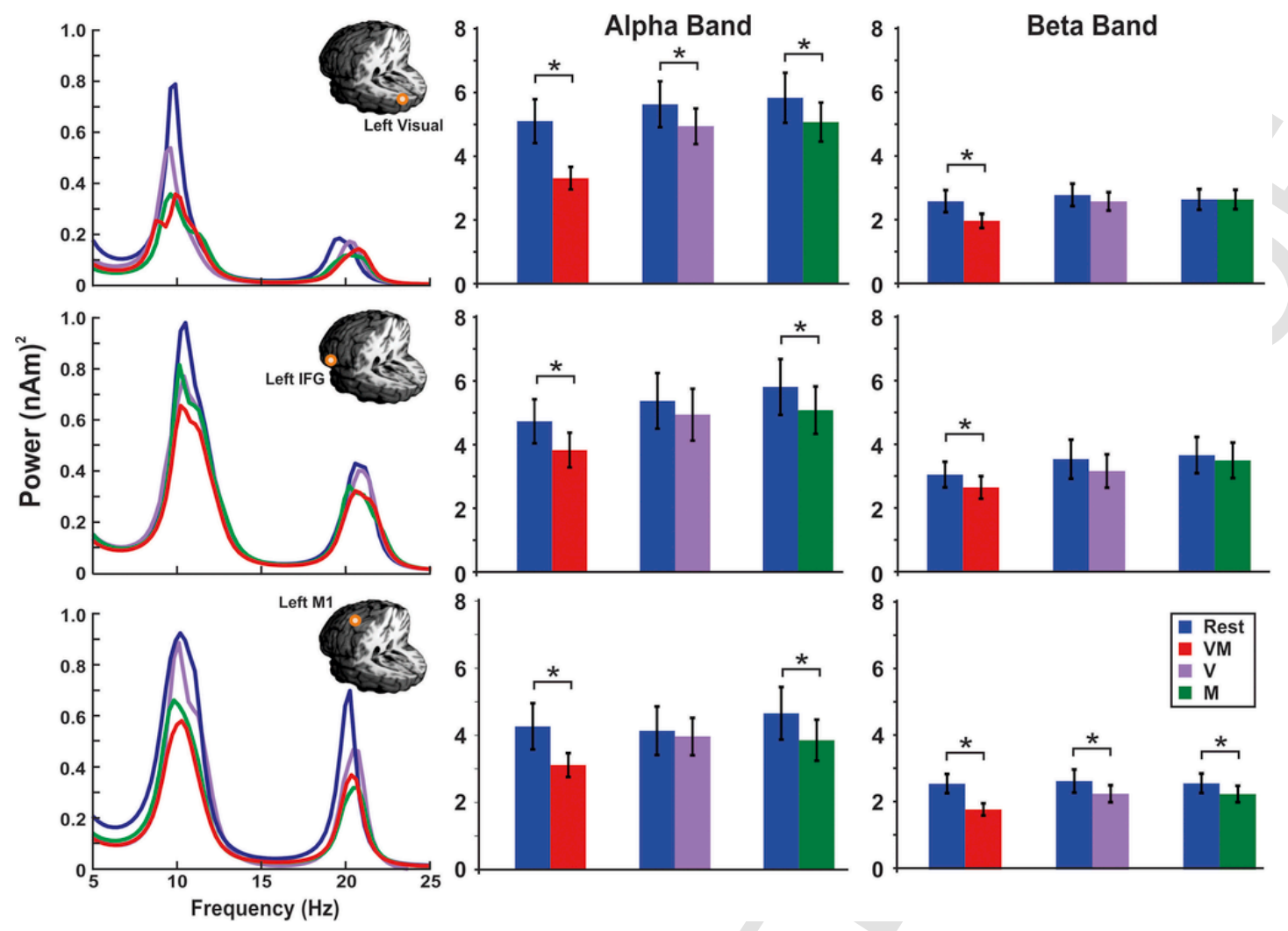

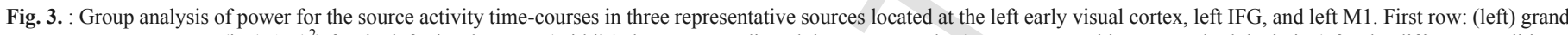

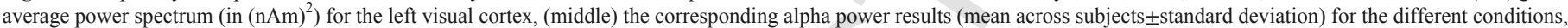

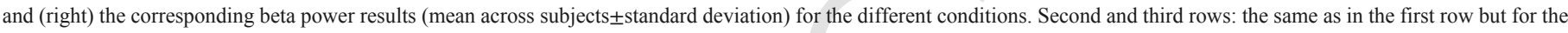
left IFG and left M1 respectively. Asterisks $\left(^{*}\right)$ indicate significant differences $(\mathrm{p}<0.05)$ between rest and VM, V, or M.

ing M. No significant correlations were observed for the other pairs of source brain activity either during VM or M.

\section{Discussion}

In the present study, we explored the coordination of neuronal activity between visual-related and motor-related parts of the brain during a sensorimotor integration task as well as during control conditions, which involved pure motor or pure visual tasks. The sensorimotor integration is a brain process that allows, by complex neural operations, the execution of a certain voluntary motor behavior in response to specific demands of the environment (Machado et al., 2008). Our sensorimotor task is a good example of functional cooperation between visual and motor areas, because it poses a demand on the brain to continuously integrate the analysis of a visual signal and produce as feedback a specific force. The two control conditions were used to test the specificity of this activity with respect to visuomotor integration process. A distributed brain network was identified that was active during this visuomotor task. This network was similar to the one frequently reported in neuroimaging studies during hand-object interaction tasks using MEG (i.e. Jerbi et al., 2007), or low-temporal resolution techniques, such as positron emission tomography (PET) (Rizzolatti et al. 1996), functional magnetic resonance imaging (fMRI) (Ehrsson et al. 2000; Castiello, 2005; Castiello and Begliomini, 2008) and transcranial magnetic stimulation (TMS) (Olivier et al., 2007). The network was comprised of the M1 contralateral to the active hand, bilateral early visual processing areas, the posterior parietal cortex around the intraparietal sulcus (IPS), a parietal opercular region most likely representing SII, and two dis- tinct prefrontal regions, one more posterior in the caudal IFG located in BA45, and a separate one located around the most anterior portion of the inferior frontal sulcus (IFS). In all these areas, an ERD was observed in the alpha and beta frequency band during VM compared to rest. Our results extend the findings of previous EEG studies that identified the brain network involved in very similar integrative visuomotor behaviors by providing the exact location of the active brain regions (Classen et al., 1998; Rilk et al., 2011). The communication between the primary visual (BA18) and the primary motor cortex (BA4) (the first and last steps of the brain network involved in visuomotor integration) was achieved through a synchronization mechanism of functional coupling in the alpha band that involved two areas of the frontal cortex (the left anterior IFS and the right IFG). This functional coupling mechanism was specific to the $\mathrm{VM}$ that required visuomotor integration, since it did not occur in the two control tasks that involved pure visual or pure motor processes.

\subsection{Activity in primary sensory and motor areas during VM and control tasks}

We observed an alpha and beta ERD during the VM task in M1 (BA4) and in early visual areas (BA18). ERD is associated with increased excitability of the cortical neurons during sensory, cognitive, and motor processing (Gastaut, 1952; Neuper and Pfurtscheller, 2001), and linked with motor-related brain functions (Pfurtscheller and Lopes da Silva, 1999; Pfurtscheller et al., 2006). Brain activity within the M1 contralateral to the active hand was expected since this area plays a fundamental role in the execution of skilled manipulatory tasks especially those that involve a precision pinch (Kuypers, 1981; 
Alpha Band
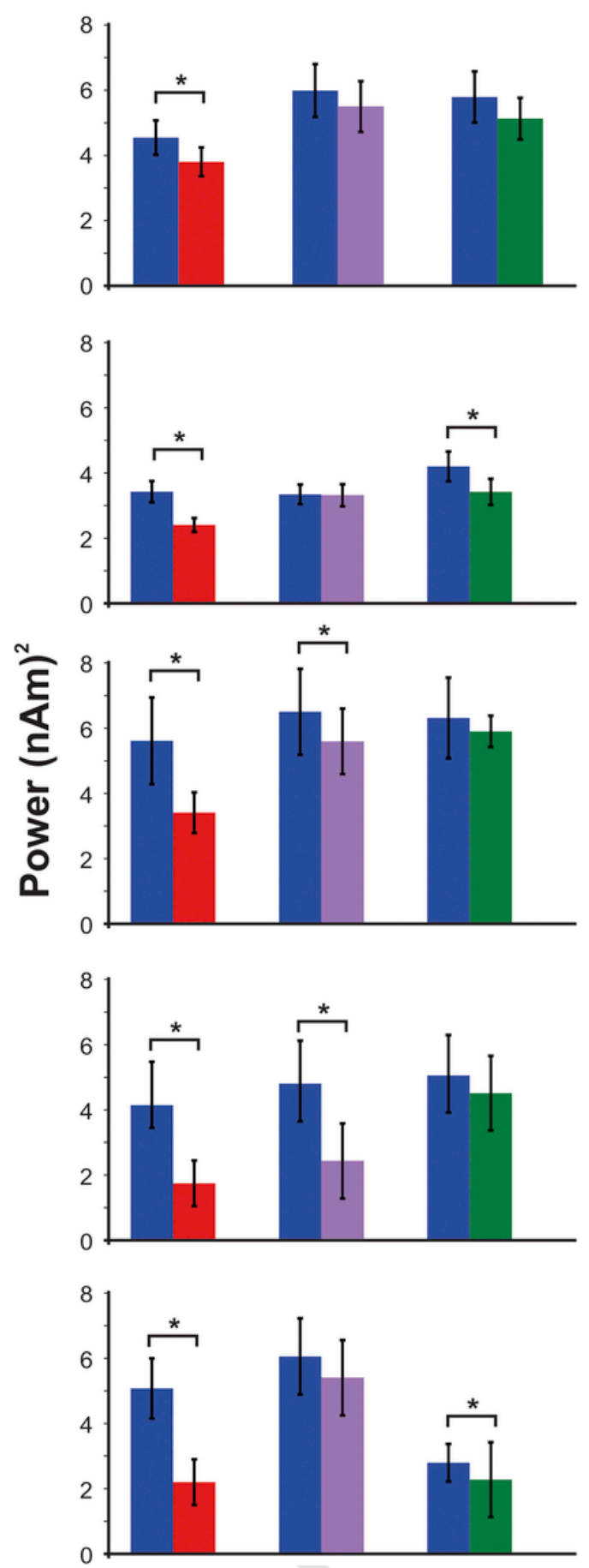

Beta Band

Right IFG

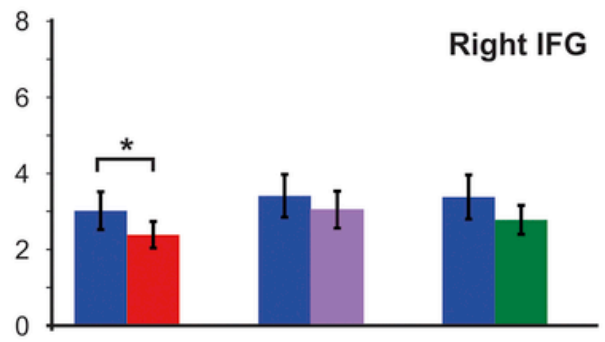

Right SPL

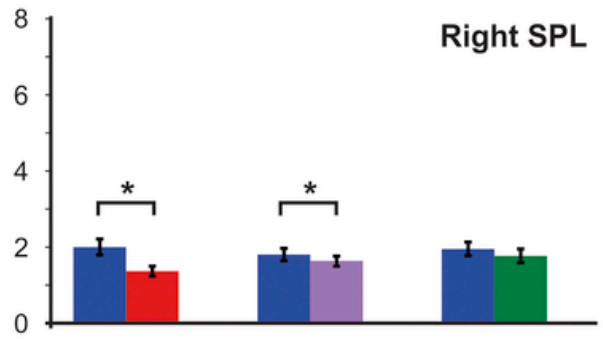

Right Visual
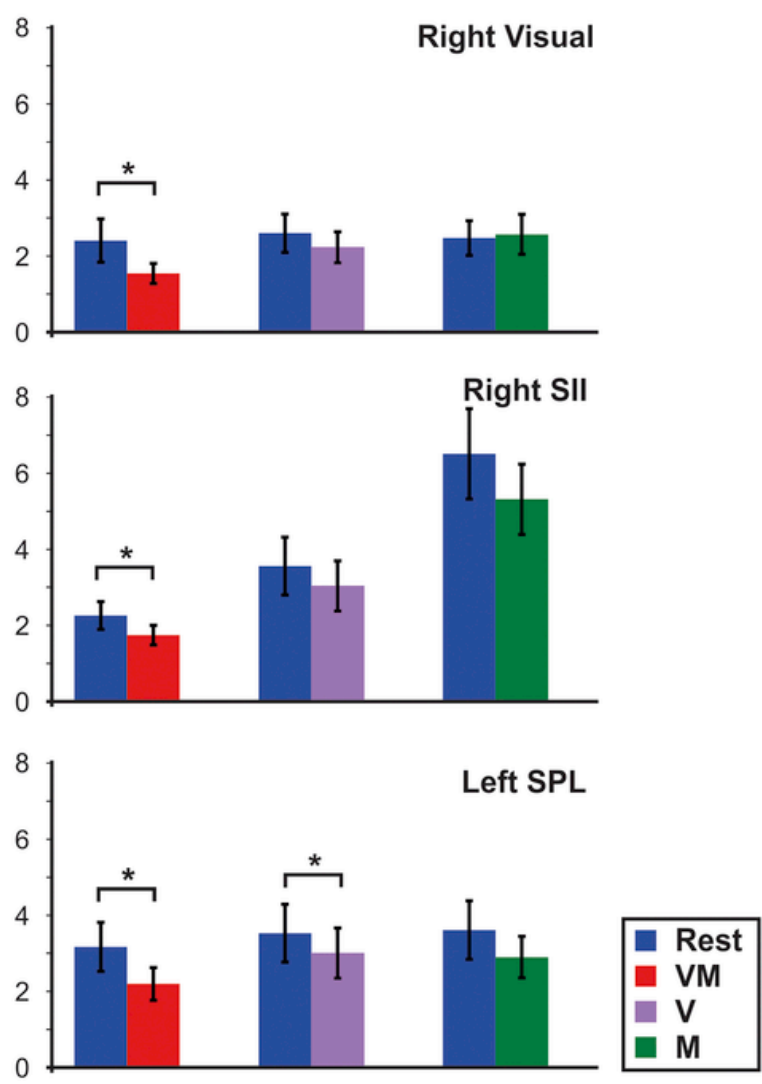

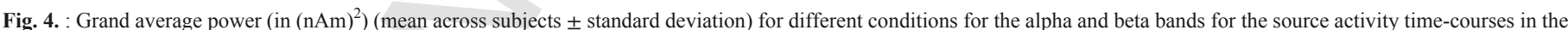

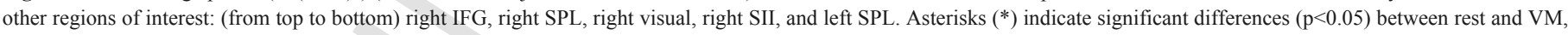
$\mathrm{V}$, or M.

Porter and Lemon, 1993). Studies in non-human primates have indicated that M1 is of utmost importance for the control of force output during precision-pinch tasks (Cadoret and Smith, 1995; 1997; Hepp-Reymond, 1988; Maier et al., 1993; Picard and Smith, 1992). Previous neuroimaging studies in humans have also shown that M1 is tive during many types of voluntary hand movements (Roland and Zilles, 1996) including isometric contractions (Salenius et al., 1997).

Despite the fact that the VM task required also proprioceptive inputs, our source localization findings did not reveal activity in the primary somatosensory cortex. This may be due to the limited ability of the beamformer to separate sources which are located close to each 

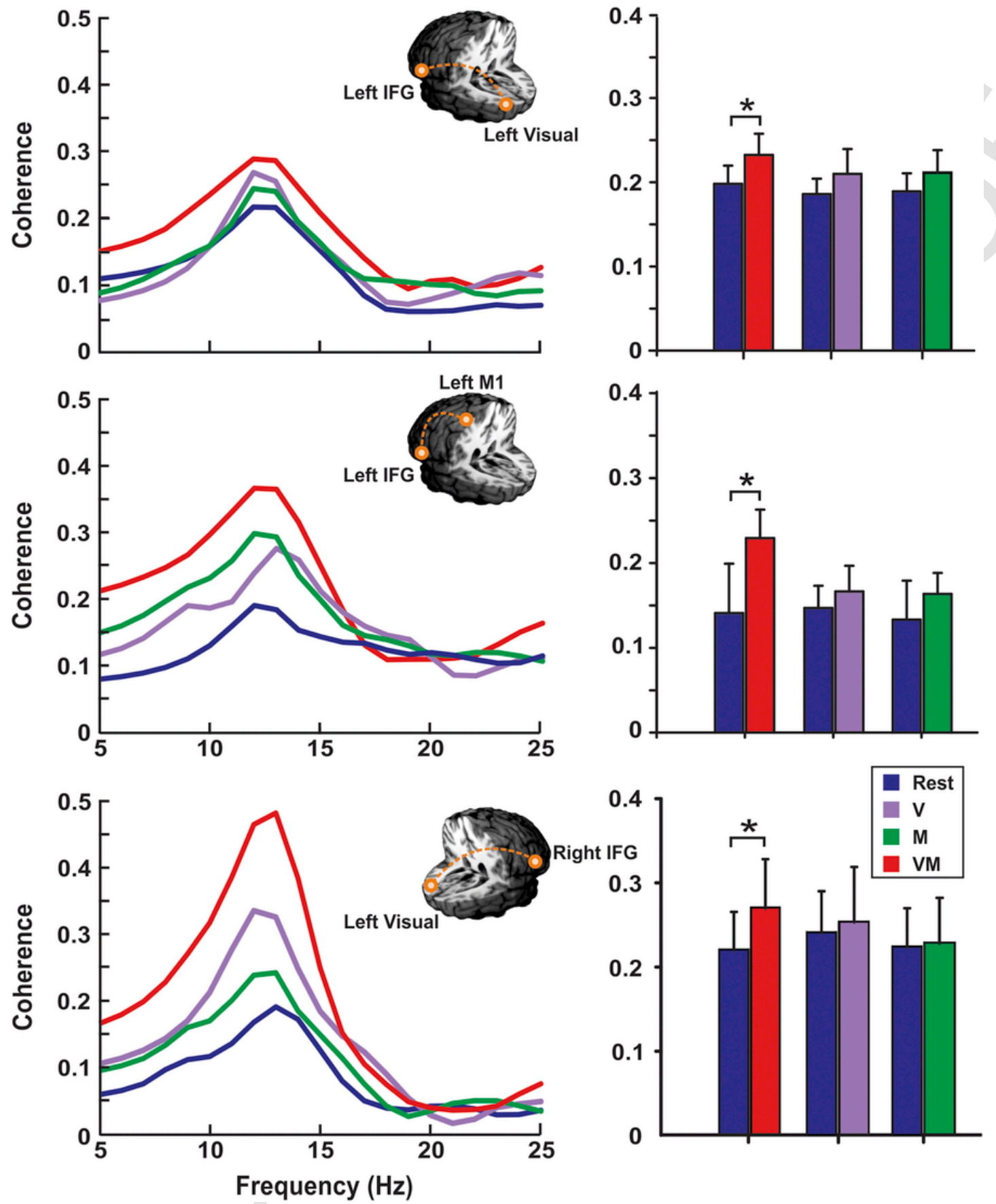

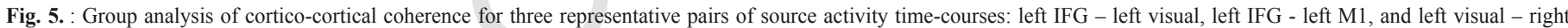

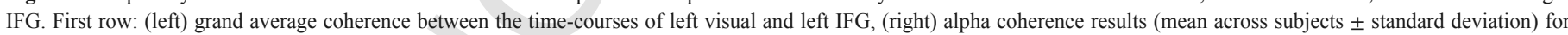

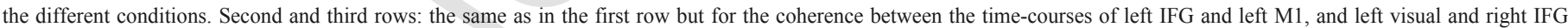
respectively. Asterisks $(*)$ indicate significant differences $(\mathrm{p}<0.05)$ between rest and $\mathrm{V}, \mathrm{M}$, or VM.

other. Thus, the active source in BA4 may actually reflect brain activity from two neighboring sources, one motor and one somatosensory, which the beamformer was not be able to discriminate. The ERD in the left M1 was in both alpha and beta bands during VM. Alpha ERD in the left M1 was specific to the two tasks that involved motor control (i.e. VM and M) (see Fig. 3). This is in line with previous EEG studies examined task-related power changes during similar visuomotor tasks (Chen et al., 2003; Rilk et al., 2011). Alpha ERD in the sensorimotor cortex is a consistent finding of motor processing in MEG and EEG studies (for a review see Cheyne (2012). It is actually characteristic for activated cortical areas ready to process information or to prepare for an action (Pfurtscheller et al., 1997). According to the theory of gating by inhibition (Jensen and Mazaheri, 2010), the alpha ERD in the sensorimotor cortex may designate the transition of this cortical area from an 'inactive' to an 'active' state. This process may not be related per se to the details of the information being processed, but to the general state of large-scale sensorimotor activation (see Kelso et al., 1998; Chen et al., 2003). Excitation of M1 for the alpha 


\section{Left IFG}
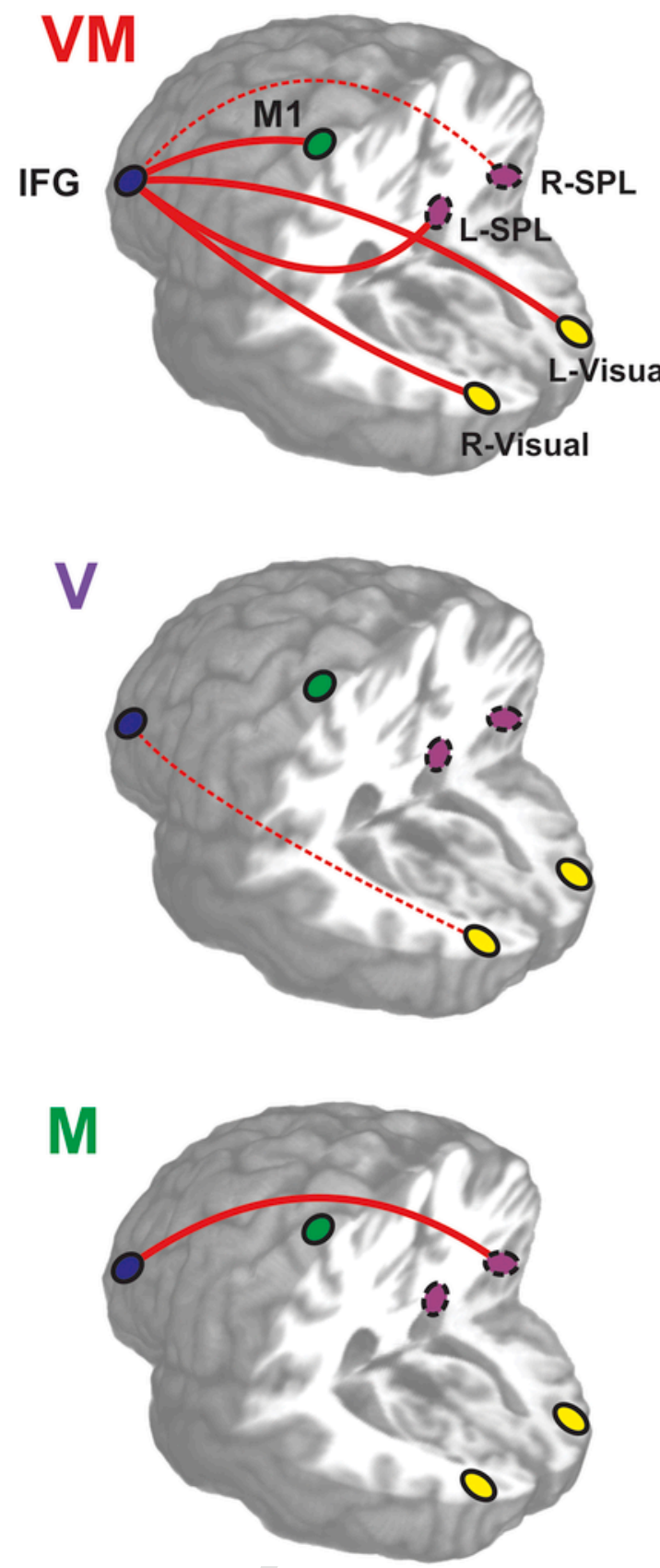

Right IFG
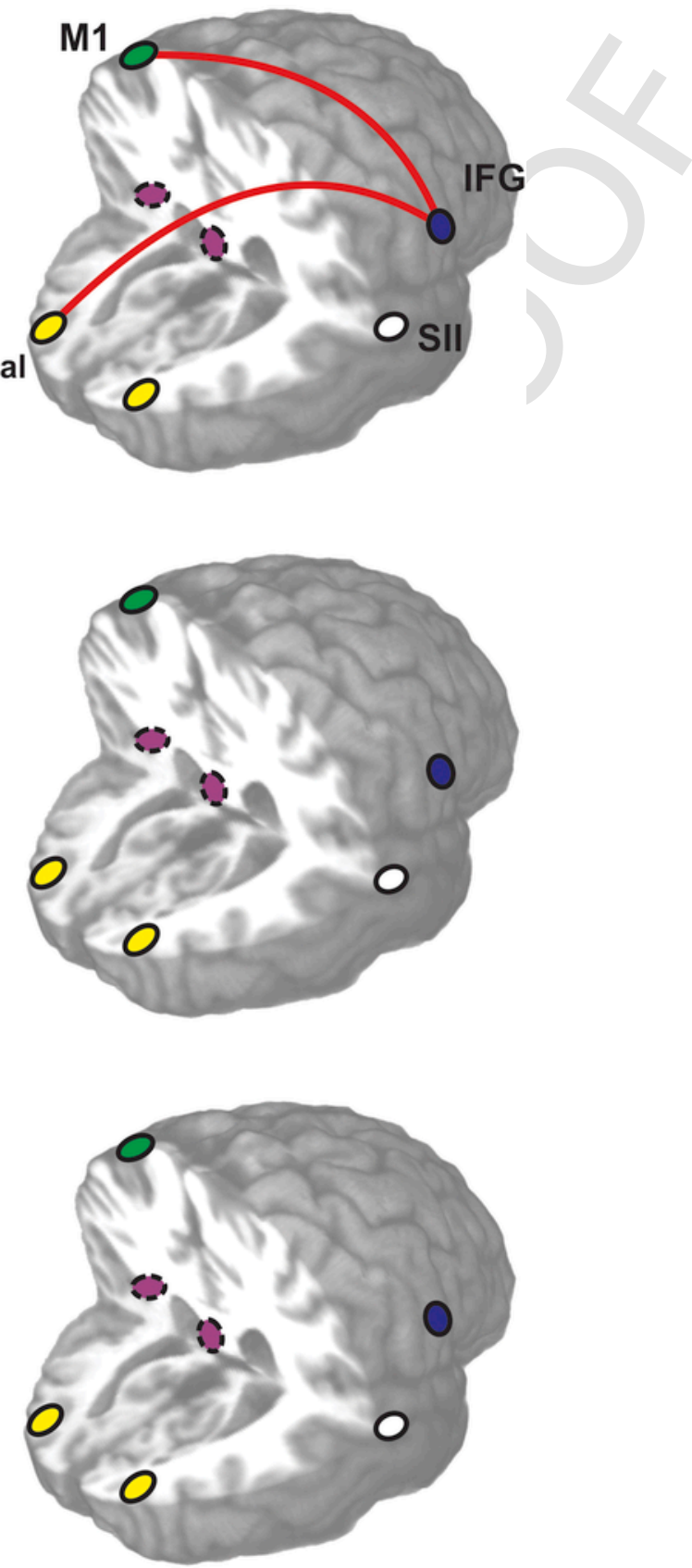

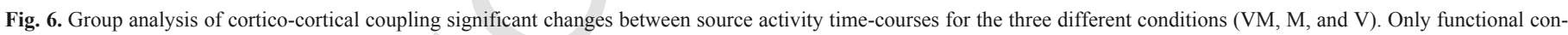

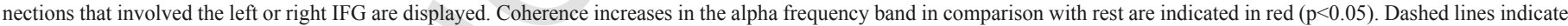
functional coupling which were marginally significant.

frequency band was observed for both VM and $\mathrm{M}$ tasks that both involved movements and not for the pure visual (V) task; thus M1 excitation in alpha band can be attributed to motor action and was not specific to visuomotor integration.

The most prominent source of beta-band ERD in relation to motor action is in the contralateral peri-Rolandic region (Pfurtscheller and Berghold, 1989; Sanes and Donoghue, 1993; Murthy and Fetz, 1996; Pfurtscheller and Neuper, 1997; Schnitzler et al., 1997; Formaggio et al., 2008). However, beta ERD can also be found in other areas across the motor-related brain network (Klostermann et al., 2007;
Courtemanche et al., 2003; Alegre et al., 2005; Paradiso et al., 2004; MacKay and Mendonca, 1995; Brovelli et al., 2004). In our study, beta ERD was observed in the contralateral M1 but it was not specific to motor-related tasks; it was observed for all three tasks. This is in contrast with the traditional view according to which beta oscillations are specifically related to motor control, but in line with some recent neuroimaging studies showing that beta oscillations are not specific and little informative to the decoding of the cognitive aspects of a motor action (Rickert et al., 2005; Pistohl et al., 2012). One source of this controversy may relate to the fact that beta modulations in differ- 


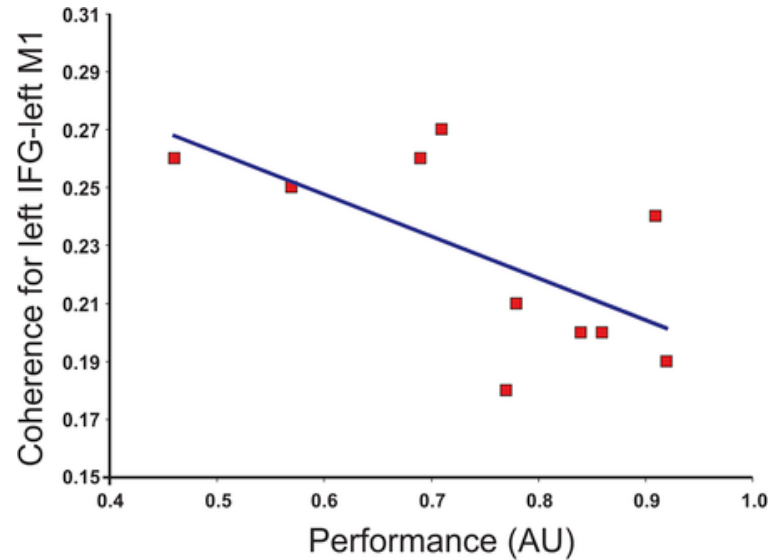

Fig. 7. Correlation of coherence in the alpha band (between the left IFG and left M1) and the performance (arbitrary units).

ent studies are often analyzed in different time windows, before, during or after a motor action (Zaepffel et al., 2013). In a classical evoked response paradigm, a motor action is preceded by a beta-band ERD with a gradual decrease of power that reaches a minimum during the action (Kristeva-Feige et al., 1993; Stancàk and Pfurtscheller, 1996) and it is followed by a phasic ERS after the termination of the action (Stancàk and Pfurtscheller, 1996; Salmelin et al., 1995). Here, we focused mostly on ongoing cortical activities since our goal was to assess the functional synchronization between distinct brain areas during visuomotor integration. Thus, our experimental setup did not follow an event-related approach that allows the clear discrimination between movement preparation, execution, and post-movement period. This may explain the fact that beta oscillations appear to be non-motor specific in our findings. Another possible explanation has to do with the fact that the effect of attention was not addressed. Indeed, all our tasks (even the control conditions) were attention demanding and desynchronisation in the beta range is known to be activated by attentional demands (Jantzen et al., 2001; Chen et al., 2003; Mayville et al., 2001).

An ERD was also observed in early visual areas for both alpha as well as beta frequency bands during the VM task. An alpha ERD was observed in the visual cortex during all tasks, while a beta ERD in the visual cortex was specific to the VM task. The alpha ERD in the visual cortex during purely motor or visual tasks confirms previous findings by other investigators (Pfurtscheller and Aranibar, 1977; Pfurtscheller and Berghold, 1989; Pfurtscheller et al., 1994) according to which any kind of visual stimulation will result in a short-lasting desynchronization of brain rhythms within the upper alpha band (10-12 Hz) focused over occipital areas (Pfurtscheller et al., 1994).

\subsection{Activity in secondary integration areas during VM}

Except activity in primary motor and sensory areas (i.e. M1 and V1), we also observed activity in two distinct prefrontal regions (left and right IFG) and two areas in the posterior parietal cortex (left and right SPL) during VM. Out of these regions, only the IFG was specifically active during VM and not during control conditions. This observation together with previous findings from fMRI studies (Ehrsson et al., 2000) indicate the specificity of this brain region to the production of skilled forces by an isometric precision pinch during sensorimotor integration. Indeed, the lateral prefrontal cortex (here right IFG) is particularly engaged in skilled force production with a high degree of sensorimotor control, such as in the precision grip (Ehrsson et al., 2000). Neuroimaging and neuropsychological evidence have shown that the caudal IFG plays a general role in motor control by interfacing external information with an internal motor representation of hand and arm actions (for a review see (Binkofski and Buccino, 2006)). Activity in rostral IFG is consistently seen in visuomotor tasks in which a continuous switching of behavior is required by the task rules, in line with continuously changing external feedback (Meiran et al., 2000). The activity in the rostal IFG could also represent a component of an error-monitoring network that was active during the VM task (Rabbitt, 1966). Our task indeed involved continuous unpredictable alternations between the two opposite behaviors of squeezing or relaxing the thumb and the index grip. In order participants to maintain a good performance as they were instructed, their brain should continuously monitor the committed errors and immediately modulates the output to generate adequate behavioral adjustments.

Previous studies in humans and animals indicate that the premotor cortex is involved in processing the visual information necessary for motor planning (Iacoboni et al., 2004; Iacoboni and Zaidel., 2004). Dorsal premotor neurons have been shown to have both attentional, gaze-related activity and intentional, limb movement activity in response to visual targets (Boussaoud, 2001; Boussaoud and Wise, 1993; Jouffrais and Boussaoud, 1999; Wise et al., 1992,1996). More recently, Sugawara et al. (2013) observed with MEG premotor activity in humans prior to visually guided movement, even for a simple movement task (index finger extension). In addition, they observed activity in the premotor cortex even during interstimulus intervals (intervals between the visual cue and the motor action) that may represent a neurophysiological marker of response selection. In monkeys, numerous studies (for example, Li et al. 1997; Kotra et al. 1997) have demonstrated that premotor activity precedes movement, suggesting that this area is also involved in motor planning. Here, we did not observe significant ERD or ERS during VM compared to rest. It is unclear why the premotor cortex was not active in our data. One possible explanation is that our experimental design and beamformer analysis canceled out the activity because they compared active versus baseline conditions based on the onset of the VM task. The onset was defined by the performance measure. Activity in the premotor cortex often precedes the movement onset. This activity was included here in the baseline condition of the beamformer analysis. However, since the VM task involved continuous unpredictable finger movements, the premotor cortex may be also active during the VM task (active condition of the beamformer analysis). Thus, the differential images of the beamformer, which compared active versus baseline conditions, were possibly cancelled out the brain activity in the pre-motor cortex.

\subsection{Functional coupling between activations in visual and motor cortex through IFG}

Our results indicate a functional hub subserving visuomotor integration that linked the early visual and primary motor areas through the left anterior IFS node and the right IFG of the network. Such coherent oscillatory activity was specific to the VM task. It did not occur in the control conditions that involved pure visual or pure motor processes and did not require visuomotor integration (see Fig. 6). These results indicate that relatively elementary visual features may be able to reach the prefrontal cortex straightforwardly. This is not surprising if we consider findings from recent animal studies that showed activation in prefrontal sites during visuomotor integration, as early as in the striate cortex (Ledberg et al., 2007). Such findings suggest that the passage of the feedforward sweep through the full cortical visual hierarchy may not be obligatory for the activation of 
frontal cortex by a visual stimulus. This functional connection may be complementary to the traditionally proposed step-by-step engagement of the dorsal stream in an occipital-parietal-premotor-motor pathway expected in a visuomotor manipulation task (Jeannerod et al., 1998). A known anatomical substrate in humans that can mediate this functional coupling could rely on the inferior occipito-frontal fascicle (IOFF). The IOFF extends from the dorsal and medial parts of the occipital lobe to the dorsal and medial part of the prefrontal regions (Makris et al., 2007), and allows the communication between extrastriate areas and areas of the frontal cortex. However, it is important to note that our findings of cortico-cortical coherence are exquisitely functional in nature and cannot by any means be considered as evidence for direct anatomical connections. More broadly, coherence between two oscillators does not provide any information about the source of synchronization. The activity may either be driven by one of them, or the two sources could be synchronized by a third deeper source.

\subsection{Functional coupling in the alpha frequency band}

The neuronal assemblies at the distributed brain sites were joined together oscillating in alpha frequency band. Our results extend the findings of a previous EEG study which used the same experimental paradigm and was limited by the poor spatial resolution of EEG (Rilk et al., 2011). In this previous study, Rilk et al. (2011) observed enhanced interregional coherence in the alpha band between contralateral frontal and central areas (i.e. areas covered by the EEG electrodes: F3, Fz, F4, C3, Cz, C4, P3, Pz, and P4). However, several findings over the past decade report different frequency bands for human brain synchronization leading to controversial attribution of information process and oscillatory activity. Some previous studies have shown synchronization in high frequency (in the gamma band) over short distances, while long range synchrony is mostly observed in the lower frequency ranges (theta, alpha, and beta) (Jerbi et al., 2007; Roelfsema et al., 1997; von Stein and Sarnthein, 2000; for review see Schnitzler and Gross (2005). Slowly oscillating cell assemblies are believed to comprise more neurons (Singer, 1993) and are likely to be spatially more extended than rapidly oscillating cell assemblies, a notion that has also been supported by modeling studies (Traub et al., 1996). Visuomotor experiments similar to our own one yielded controversial findings of coupling in different frequency bands. Jerbi et al.(2007) reported a long-range task-related coupling between the primary motor cortex and multiple brain regions (including the contralateral dorsolateral prefrontal cortex (DLPFC), the contralateral dorsal premotor, and SPL) in the frequency range of $2-5 \mathrm{~Hz}$, while Classen et al. (1998) reported synchronization among different brain regions in the alpha and beta bands during visuomotor integration by using EEG. These differences in the frequency of synchronization may be attributed to differences in the involved tasks. For example, in Jerbi et al. (2007), subjects used their palm to manipulate a round-button joystick simulating a more gross movement, while in Classen and colleagues (1998) subjects used their fingers to manipulate a pinch grip.

\subsection{Correlation between task performance and coherence of brain activity}

Our data indicate an inverse relation between the strength of prefrontal-motor oscillatory coherence and the efficiency in the VM task. This means that the functional connectivity between the IFG and the M1 was larger when larger was the generated force in the pinch force task. This finding suggests the prefrontal-motor network to play a functional role as an online performance monitoring system that operates through a local prefrontal-motor link. Performance monitoring is a key component in our VM task; it concerns the identification and correction of differences between an intended and an executed response. Recent mapping human studies have identified the spatial and temporal components of such a putative performance monitoring system in the large-scale networks of the human brain. This system involves different brain areas in the frontal and prefrontal cortex, such as the medial frontal cortex, the anterior insula/inferior operculum, and the lateral prefrontal cortex (Garavan et al., 2003; Hester et al., 2005; Kiehl et al., 2000; Menon et al., 2001; Polli et al., 2005; Rubia et al., 2003). The role of the ventrolateral prefrontal cortex (consisted of BA45, 44, and 47) as component of this system was highlighted by recent fMRI studies see Fig. 2 in Taylor et al. (2007). Error-related activity from healthy subjects was reported bilaterally in the ventrolateral prefrontal cortex, while subjects performing an interference task. Here, the left IFS and right IFG may indicate active nodes of this performance monitoring process. Inverse correlation of the performance with the prefrontal-motor oscillatory coherence may indicate the final stage of the executive control of this process exerted by the prefrontal cortex on the motor system. This notion is further supported by previous findings showing that prefrontal function requires the sending of feedback signals to reach widespread targets throughout the brain (for a review see Miller and Cohen (2001)).

\subsection{Methodological considerations}

Although MEG experiments that involve movements always face the problem of possible contamination of brain activity signals with muscular activity, we are confident that in our data such a contamination was negligible for the following reasons: (i) our analysis focused on brain activity with frequencies from 1 to $30 \mathrm{~Hz}$ (the data were filtered in this frequency band); muscular activity is at higher frequencies well-above $30 \mathrm{~Hz}$; (ii) a beamformer was used to localize the brain activity; beamformer-based source localization techniques provide an efficient account for cranial muscle and saccadic spike artifacts in addition to high spatial specificity in the source space Hipp and Siegel (2013); (iii) special care was taken that the participants sat comfortably in order to reduce postural muscle artifacts and relax their facial muscles, and (iv) trials contaminated with muscle artifacts, signal jumps or distortions of the magnetic field were rejected off-line using automated threshold procedures applied to the MEG signals. An improved experimental design would have included the recording of electromyographic activity during all three tasks.

\section{Conclusions}

In summary, our study discloses for the first time the neurophysiological patterns of intraregional and interregional oscillatory activity related to sensory-motor integration in a visuomotor precision pinch force task at the source level. Previous EEG studies also tried to identify the brain network involved in similar integrative visuomotor tasks (Classen et al., 1998; Rilk et al., 2011), but the analysis was performed at the sensor level and the location of the active brain regions was not assessed. Here, we identified with MEG a complex large-scale neural network that was active during visuo-motor integration. The network was comprised of primary sensory areas, such as the primary visual cortex, the primary motor cortex contralateral to the active hand, and secondary integration areas. This network allowed the functional integration of motor areas with areas processing sensory information through a neural synchronization mechanism in 
the alpha frequency range. Our findings add to the existing knowledge about visuo-motor integration by revealing the importance of the left rostral and right IFG as functional hubs that subserve visuomotor integration during performing a visuo-motor precision pinch force task with the right hand. The findings indicate a functional occipito-prefronto-motor network for the selection of appropriate actions on the basis of determinate rules. The network may be complementary to the traditionally proposed step-by-step engagement of the dorsal stream in an occipital-parietal-premotor-motor pathway expected in a visuomotor manipulation task. The practical implications of our results include the interpretation of several aspects of neurological disorders, ranging from the basic symptoms of the prefrontal syndrome, up to the complex manifestations of neuropsychiatric disorders that produce repetitive and stereotyped behaviors such as autism.

\section{Material and Methods}

\subsection{Participants}

Twelve healthy right-handed volunteers (mean age: $25.23 \pm 2.23$ years, 6 females) participated in the study. None of the participants had a history of neurological disorder or used drugs active on the central nervous system and all of them had normal or corrected to normal visual acuity. All participants gave informed consent according to the Declaration of Helsinki and received monetary compensation for their participation. The study was approved by the ethics committee of the Medical Faculty of the University of Tübingen. We requested the participants to abstain from alcohol and caffeine the day before and the day of the experiment. The participants were informed that they could terminate the experiment at any time without the need to provide any justification for their decision (no one did).

\subsection{Experimental setup}

The experiments were performed at the MEG Center of University of Tubingen, Germany. Participants were seated comfortably in an armchair during the experiment inside a light dimmed magnetically shielded room (MSR). A MEG-compatible screen was placed at $\sim 100 \mathrm{~cm}$ in front of the participants. During the experiment, participants were asked to fixate on a stationary cross in the middle of a screen placed $\sim 100 \mathrm{~cm}$ in front of them (Fig. 1a). Participants were also asked to refrain from blinking as much as possible during the recordings. The participants' right arm was comfortably relaxed on a hand rest (Fig. 1a). By using their right hand's thumb and index fingers, participants were able to manipulate a pinch force transducer (Fig. 1b - left) that was designed and constructed at the University of Tübingen. By using strain gauges affixed to the upper and lower surface of a manipulandum, the transducer was translating the manual force applied, isometrically by the participant into the width of a visual target projected on the screen (Fig. 1b). The visual feedback was presented in the middle of the screen by using a video projector (PLC-XP41, Sanyo) standing outside the MSR. The beam of the projector entered the MSR through a cut-away portal in the shield. The resolution of the screen was 1024 by 768 pixels and the monitor frame rate was $60 \mathrm{~Hz}$. The extreme positions of the visual target projected onto the screen subtended a visual angle of $4^{\circ}$. A stationary red cross was overlaid on top of the visual target in the middle of the screen.

\subsection{Experimental design}

Our design was based on experimental paradigms used in previous studies (i.e. Classen et al., 1998; Rilk et al., 2011). In our experiment, participants should perform three tasks: the visuomotor (VM) task, the motor (M) task, and the visual (V) task. The order of the tasks was randomized between participants. Each participant performed three blocks in total: one for each task (VM, M and V) (Fig. 1c). The duration of each block was 10 min and $30 \mathrm{~s}$. Each block consisted of three trials and each trial lasted $3 \mathrm{~min}$ and $30 \mathrm{~s}$. Each trial started with $1 \mathrm{~min}$ of baseline recording $(\mathrm{R})$ during which the participants fixated the stationary cross without performing any motor task. Then subjects performed the task (VM, M, or M) for $2 \mathrm{~min}$. The task was followed by $30 \mathrm{~s}$ interval during which the participants could relax their eyes from fixation and blink if necessary (Fig. 1c).

In the VM task, participants continuously tracked the visual target stimulus appearing on the screen. Continuous tracking was achieved by adapting the manual force of the pinch grip. The force of the isometric contraction was measured by the force transducer and was linearly translated into the width of a white rectangle. A black square was also shown on the screen overlaid the white rectangle (Fig. 1b right). The width of the black rectangle was continuously changing sinusoidally with time, with a center frequency of $0.3 \mathrm{~Hz}$. The changes could be predicted by our subjects only to a certain degree. The participants were instructed to continuously align the white rectangle and the black square as precisely as possible. In the M task, the participants were instructed to imagine a metronome implying a pulse every other second and press the pinch correspondingly with half the amount of their maximum force, while both rectangles remained stationary and aligned on the screen. In the V task, the participants watched the sinusoidally varying target and the stationary stimulus without performing the precision pinch force task. To record brain activity during stationary performance and to avoid learning effects across sessions, participants were familiarized with the three tasks in a separate session preceding the MEG recordings.

\subsection{MEG and MRI recordings}

Whole-head MEG recordings were performed using a 275-channel axial gradiometer CTF system (VSM MedTech Ltd) sampled at $585.93 \mathrm{~Hz}$ and band-passed at 0-200 Hz. In total, 36 datasets of MEG data were collected (three datasets for each participant: VM, M, and $\mathrm{V})$. Before the experiment, we made sure that the participants were positioned comfortably in order to reduce postural muscle artifacts. We instructed the participants to avoid movements, eye-blinks and eye movements during the recordings and to focus on the fixation cross.

Each participant's head was also scanned with a high-resolution anatomical MRI (1.5 T MRI, Model ExcelArt, Toshiba Medical Systems) using a T1-weighted volume acquisition sequence resulting in a voxel-size of $1 \times 1 \times 1 \mathrm{~mm}^{3}$. To enable coregistration of MEG results with anatomical brain scans, three coils were placed at the nasion and pre-auricular points prior to MEG data acquisition. The coils at the fiducial localization served to continuously monitor the position of the subjects' head relative to the MEG sensors. After each acquisition, the coils were removed and at their locations, radio-opaque washers were placed carefully for off-line coregistration of the recorded MEG data with the individual structural MR images obtained from each subject. EEG data were also collected but will be presented elsewhere. 


\subsection{Performance}

Performance was quantified for the VM task as the continuous tracking error calculated by the root mean square (RMS) of the deviation of the exerted force from the requested force. Accordingly, larger differences (higher tracking error) correspond to lower performance. Calculations were based on arbitrary units (AU).

\subsection{Data pre-processing}

Standard CTF software was used to filter the MEG data by using a $50 \mathrm{~Hz}$ notch filter (and its harmonics), and by removing the DC offset. Raw data were then inspected offline for bad sensor recordings (four bad channels on average). Trials contaminated with muscle artifacts, signal jumps or distortions of the magnetic field were rejected off-line using automated threshold procedures applied to the MEG signals. MEG data from two subjects were excluded from further analysis due to heavy artifact contamination from muscle activity. The remaining trials were kept for further analysis since none of them had field magnitudes greater than $1 \times 10^{-11}$ Tesla in any channel.

\subsection{Data analysis overview}

We set out to investigate the patterns of local and interregional oscillatory activity related to the three tasks (VM, V, and $\mathrm{M}$ ) by using power and coherence estimates. We focused our analysis on the spectrum between 1 and $30 \mathrm{~Hz}$ because visuomotor integration has been associated with changes in lower frequency bands (i.e. mostly alpha and beta) (Classen et al., 1998; Rearick et al., 2001; Deiber et al., 2001; Kranczioch et al., 2008; Rilk et al., 2011). We estimated task-related oscillatory power changes compared to rest in terms of event-related synchronization (ERS) or event-related desynchronization
(ERD) (Pfurtscheller, 1992). These changes indicate intra-regional activity of localized neuronal assemblies. The ERS and ERD were estimated at the source level with a beamformer technique (see Section 5.9). The MEG data were initially filtered in the frequency band from 1 to $30 \mathrm{~Hz}$. Then, differential beamformer images were created by subtracting baseline periods (at rest) from active periods (during performing the VM task). This resulted in high resolution three-dimensional differential images, which were time-locked to the VM task onset. For each participant, these images were averaged to identify peak activation sites in the brain during the active period relative to baseline. Thus, for each voxel location in the brain, we estimated the task-related power change between the baseline and the active state during VM task. We defined as ERS, an increase in the power of the brain activity during the active (compared to the baseline) state in the frequency band between 1-30 Hz. We defined as ERD, a decrease in the power of brain activity during the active (compared to the baseline) state in the same frequency band. Statistical analysis was performed at the voxel level by using a multi-subject one sample $t$-test design. At brain locations where the ERS or ERD were statistically significant, we estimated virtual sensors (see Section 5.11). For these locations, the ERS or ERD were estimated for every task (VM, M, and V). Task-related coherence was also estimated for all pairs of virtual sensors. Coherence reflects inter-regional functional coupling of oscillatory neuronal activity. From the combination of both estimates, a comprehensive picture of sensorimotor information processing can be obtained. A schematic overview of the data analysis flow is presented in Fig. 8.

\subsection{Forward model estimation}

In order to solve the forward problem, we used a sensor-weighted overlapping-sphere head model (Huang et al., 1999). This model is regarded as a natural extension of the single sphere model (Sarvas,
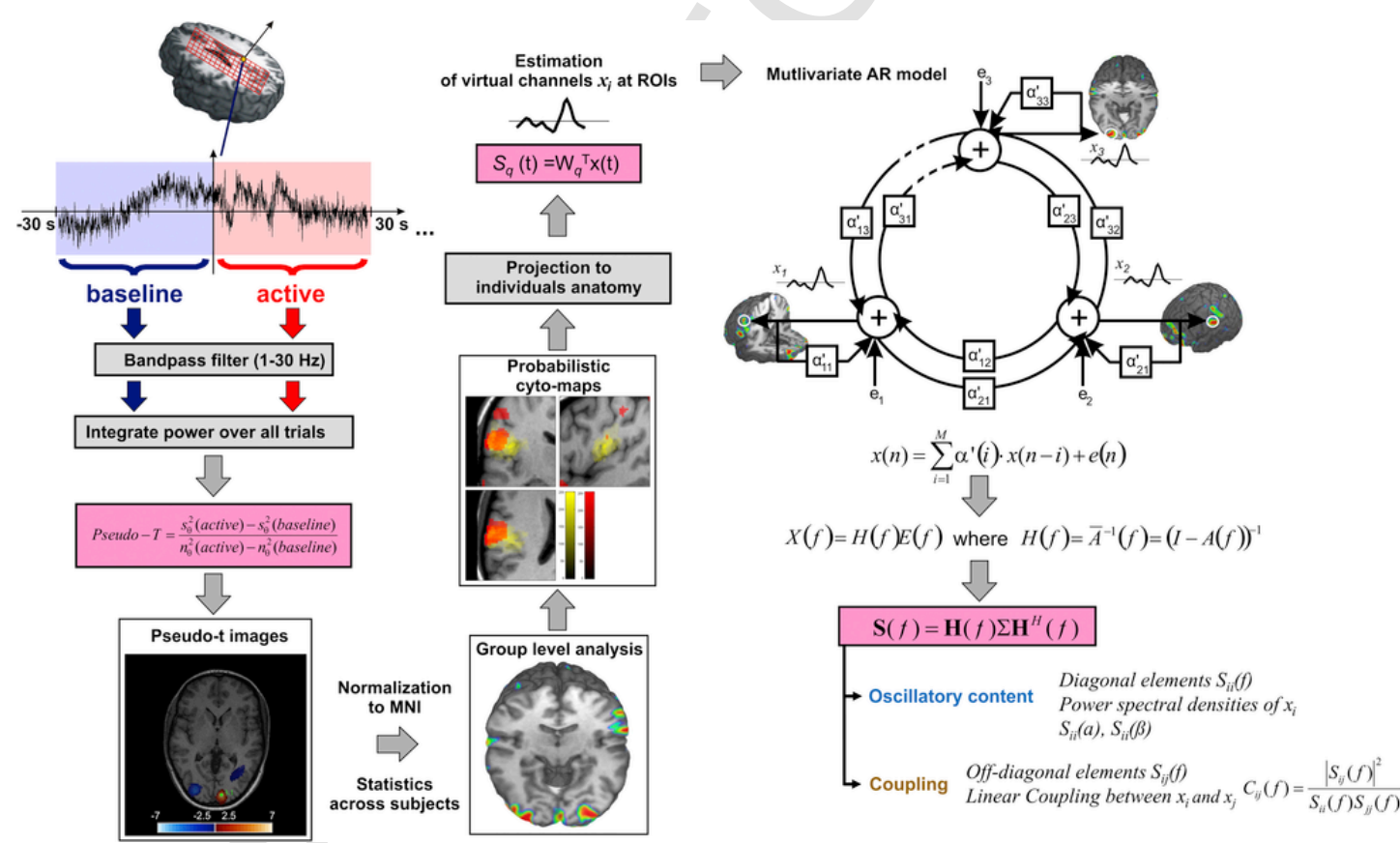

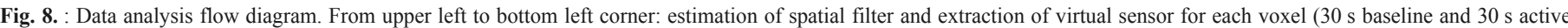

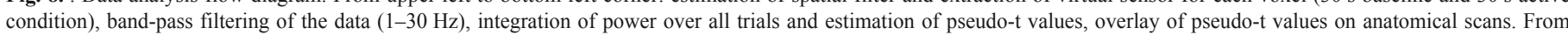

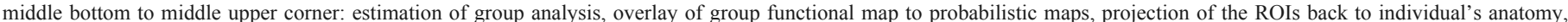

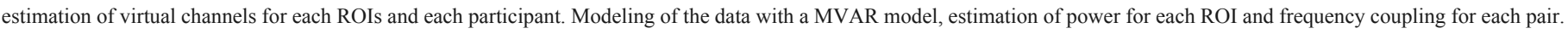


1987). The overlapping spheres model fits the spherical model on a sensor-by-sensor basis using a set of grid points within the brain. The model was estimated for each participant using their anatomical MRI. We used this head model because: (i) it is considered an improvement over single-sphere models, (ii) it is computational simpler than realistic models, and (iii) relatively simple head models are sufficient to solve the forward problem in MEG analysis (Scheler et al., 2007). The procedure is the following: (i) for a single MEG sensor, we first calculate the lead field over thousands of dipole locations using a sphere to the local curvature of the skull in the vicinity of the sensor; (ii) make an initial guess of the best fitting sphere center (for example the best fitting sphere center for the entire head) and radius, and calculate the lead field using the Sarvas formula over the same set of dipole locations; (iii) calculate the cross-correlation between these above two lead fields; (iv) adjust the center of the sphere and repeat (ii) and (iii) until the highest cross-correlation is achieved. The result is a best-fitting sphere for each sensor, yielding a set of overlapping spheres spanning the realistic head shape. More details about the estimation of the overlapping-sphere head model can be found elsewhere (Huang et al., 1999). A conductivity value of $0.33 \mathrm{~S} / \mathrm{m}$ was used in the calculations.

\subsection{Synthetic Aperture Magnetometry (SAM)}

For each participant, brain source activity was estimated for the VM task compared to rest by using the beamformer source-imaging technique called SAM (Robinson and Vrba, 1998). A beamformer is a spatial filter designed to detect a signal corresponding to a specified location and attenuates signals from all other locations. The beamformer can potentially reconstruct multiple cortical sources at any position within the brain, without any a priori spatial constraints, as long as the time courses of the sources are not perfectly correlated. One advantage of using SAM in the analysis of non-phase-locked ERS/ERD is that experimental designs can be used which do not conform to the standard evoked response paradigm.

For each voxel location in the brain, a unique set of sensor coefficients (or beamformer weights) are estimated. These coefficients are derived from the covariance matrix representing the pairwise relations of the activities at all channels. More details about the covariance matrix estimation can be found in Cheyne et al (2006). Then, the raw data are multiplied by the corresponding coefficients for each voxel, and the activity of a virtual sensor is estimated for each voxel location (see upper left corner in Fig. 8). Here, we employed the dual-state SAM imaging, which estimates the task-related power change between the two time windows: the baseline and the active. As baseline and active time windows were respectively selected the $30 \mathrm{~s}$ preceding and the $30 \mathrm{~s}$ following the onset of the VM task for each trial (see Fig. 8). The onset of the VM task was defined by the performance measure. When the first derivative of RMS was greater than one then a trigger was indicating the onset of the task (see Fig. 1d). The MEG data were initially band-pass filtered between 1 and $30 \mathrm{~Hz}$ and the power of the signal for both windows (baseline and active) was integrated over all trials. The beamformer was sequentially applied to a regular grid of locations spanning the whole brain. Then, for each voxel of each participant's MRI scan, a pseudo t-value was estimated representing the power change between the baseline and active windows normalized by the noise variance (Robinson and Vrba, 1999) (see Fig. 8). Negative and positive pseudo-t values indicate ERDs (source power decreases) and ERSs (source power increases) in the frequency band of $1-30 \mathrm{~Hz}$ respectively. The resultant volumetric maps were overlaid on the individual participant's structural MRI (see left bottom corner in Fig. 8).

\subsection{Group level analysis}

Each participant's MRI was spatially normalized into the Montreal Neurological Institute (MNI) space via the segmentation module of the Statistical Parametric Mapping 5 (SPM5) (http://www.fil. ion.ucl.ac.uk/spm/). The resulting normalization parameters were then applied to the SAM volumetric images. Hence, all of the volumetric images were then in the same 3-D coordinate space and could be carried forward into a group statistical analysis (see Fig. 8). The Statistical non-Parametric Mapping (SnPM) software was used for the group statistical analysis. More details about the application of non-parametric permutation tests on SAM analysis can be found in Singh et al (2003). Within SnPM, analysis was performed at the voxel level by using a multi-subject one sample $t$-test design. We used a nonparametric method based on permutation because it outperforms the conventional parametric test (Nichols and Holmes, 2002; Singh et al., 2003). For group analysis, non-parametric permutation tests were conducted by using the full permutation set (1024 permutations) with $10 \mathrm{~mm}$ variance smoothing. In order to correct for false positives due to multiple-testing, the Bonferroni-Holms method was used. The level of statistical significance was set at $\mathrm{p}<0.05$. The permutation test statistical results were visualized on a single subject MRI normalized in the MNI space. Group level statistical maps of the activation across participants revealed eight areas of local maxima. Spatial regions of interest (ROI) were selected at these areas of significant brain activity.

\subsection{Probabilistic cyto-maps}

We identified the significant activation sites and volumes for the VM task by using the probabilistic atlas available through the anatomy toolbox (Eickhoff et al., 2005). The use of probabilistic maps in the identification of underlying regions by using MEG has been shown for cortical (Barnikol et al., 2006; Papadelis et al., 2011) and subcortical (Liu and Ioannides, 2010; Styliadis et al., 2014; 2015) areas. We automatically assigned an identity to our activation sites based on the probabilities at the maxima of the activities. The assignment algorithm used (Eickhoff et al., 2006), is based on the assignment of each voxel to the most probable anatomical area at the position under investigation. The probability limit for assignment was set to $\geq 80 \%$. In the case that the activity could be assigned in two distinct cytoarchitectonic areas, we chose the one with the higher probability.

\subsection{Projection to individuals' anatomy and estimation of virtual sensors}

The ROIs from the group level analysis were then projected back to the individual's MRIs by using the inverse normalization process from MNI to the coordinate space of each participant. Virtual sensors were then estimated at the eight ROIs for all three tasks (VM, M, and $\mathrm{V}$ ) by multiplying the SAM weights with the raw data (see Fig. 8). Virtual sensors were defined as a weighted-sum of the sensor data that estimates the time-course of electrical activity at specific points in the source space via a spatial filter (Robinson and Vrba, 1999). We used a common spatial filter for all conditions in order the results from the different conditions to be comparable. 


\subsection{Multivariate Autoregressive (MVAR) model}

To move beyond the identification of regional activations and toward the characterization of functional circuits underpinning sensorimotor integration, we used an MVAR model. MVAR models have been proposed to make inferences about functional integration within the human brain. Their use in the analysis of neuroimaging time series is well described in the literature (Harrison et al., 2003). MVAR models quantify the linear dependence of one region upon all others in the network. The use of MVAR models for characterizing networks of cortical activity associated with cognitive tasks allows us to quantify the dependence among all possible combinations of pairs of regions in the model. In this way, connectivity architectures can be compared across different cognitive tasks.

Here, we used a MVAR model (Kay, 1988) to estimate the power and coherence of the source activity in the alpha and beta frequency bands (see upper right corner in Fig. 8). The time-courses were down sampled and normalized (zero-mean). The MEG signals of the eight virtual sensors were then described by a MVAR model (Kay, 1988) as:

$$
x(n)=\sum_{k=1}^{M} a^{\prime}(i) x(n-i)+e(n)
$$

with $M$ model order, $a^{\prime}(i)$ a matrix describing the linear interaction from $x(n-i)$ to $x(n)$, and $e(n)$ zero-mean uncorrelated white noises with diagonal covariance matrix $\sum$ (see Figure 8$)$. Model coefficients were estimated, with fixed order $p=8$, by standard vector least squares identification. Eq. (1) was transformed into the frequency domain to yield $X(f)=H(f) E(f)$, with $X(f)$ and $E(f)$ Fourier Transforms of $x(n)$ and $e(n), \quad$ and $H(f)=\bar{A}^{-1}(f)=(I-A(f))^{-1}$ transfer matrix.

The spectral matrix was obtained as $S(f)=H(f) \Sigma H^{H}(f)$. The diagonal elements $S_{i i}(f f)$, are the power spectral densities of each modeled signal $x(n)$. Multivariate spectral decomposition (Baselli et al., 1997) was applied to each spectrum $S_{i i}$ to find the partial spectra related to the poles of the process for the alpha $(8-13 \mathrm{~Hz})$ and beta $(13-30 \mathrm{~Hz})$ frequency bands. The area underlying this partial spectra was then taken as a measure of the power within the band $\bar{P}_{\alpha}$ and $\bar{P}_{\beta}$ . The off-diagonal elements of $S(f)$ were used to measure the linear coupling between each pair of signals $y_{i}$ and $y_{j}$ through the squared coherence function (Kay, 1988):

$$
C_{i j}^{2}(f)=\frac{\left|S_{i j}(f)\right|^{2}}{S_{i i}(f) S_{j j}(f)}
$$

The average coherence $\bar{C}_{i j}^{2}$ was computed for the two frequency bands.

\subsection{Statistical analysis}

The significance of the coupling between the considered series was assessed by setting a threshold level in the coherence function, using a procedure based on FT surrogate series (Faes et al., 2004). 95th percentile of the coherence distribution for the 100 generated surrogate series was adopted as threshold. The average coherence for each frequency band was then compared to the average threshold in the same band, and set to zero if lower than the average threshold. Both for power and coherence, the average value across the three trials was estimated for each subject and condition. Wilcoxon paired $t$-test were performed to assess the significance of the difference between rest and task conditions (VM, M, and V) both for spectra and coherence. The significance level was set to $\mathrm{p}<0.05$.

\subsection{Task performance and its correlation with coherence}

The correlation between the task performance (correlation between the requested and the performed pinch force) and the coherence of brain activity time-courses was estimated.

\section{Uncited references}

(Aoki et al., 2000; Binkofski et al., 1998; Bradley et al., 2000; Davare et al., 2009; Fogassi et al., 2001; Gallese et al., 1994; Goodale, 2008; Goodale and Milner, 1992; Grafton et al., 1998; Inoue et al., 1998; Kawashima et al., 1998; Konishi et al., 2005; Lebedev and Wise, 2002; Machado et al., 2010; Makris et al., 2005; Matelli et al., 1985; Matsumura et al., 1996; Milner and Goodale, 2008; Neuper et al., 2007; Papadelis et al., 2009; Pause et al., 1989; Petrides and Pandya, 2007; Ramnani and Owen, 2004; Rao et al., 1993; Rizzolatti and Luppino, 2001; Rizzolatti et al., 1998; Rowe et al., 2000; Salmelin and Hari, 1994; Seitz et al., 1997; Spraker et al., 2009; Taniguchi et al., 2000; Tomassini et al., 2007; Tunik et al., 2005; Wise, 2008).

\section{Acknowledgments}

This work was supported by the Provincia Autonoma di Trento; the Fondazione Cassa di Risparmio di Trento e Rovereto; the German Ministry for Research and Technology; and the Werner Reichardt Center for Integrative Neuroscience (CIN) at the University of Tübingen. The CIN is an Excellence Cluster funded by the Deutsche Forschungsgemeinschaft (DFG) within the framework of the Excellence Initiative (EXC 307). Data were collected by C.B., data analysis was performed by C.A. and S.E., and supervised by G.N., while the manuscript was prepared by C.P., Wise (2008), and C.B. We gratefully thank Mr. W. Kern and Mr. J. Dax who built the manipulandum and control electronics for the force measurement, and Dr. M. Tobei for his valuable comments and suggestions.

\section{References}

Alegre, M., Alonso-Frech, F., Rodríguez-Oroz, M.C., Guridi, J., Zamarbide, I., Valencia, M., Manrique, M., Obeso, J.A., Artieda, J., 2005. Movement related changes in oscillatory activity in the human subthalamic nucleus: ipsilateral vs. contralateral movements. Eur. J. Neurosci. 22, 2315-2324.

Aoki, F., Fetz, E.E., Shupe, L., Lettich, E., Ojemann, G.A., 1999. Increased gamma-range activity in human sensorimotor cortex during performance of visuomotor tasks. Clin. Neurophysiol. 110, 524-537.

Aoki, F., Fetz, E.E., Shupe, L., Lettich, E., Ojemann, G.A., 2000. Changes in power and coherence of brain activity in human sensorimotor cortex during performance of visuomotor tasks. Biosystems 63, 89-99.

Aranibar, A., Pfurtscheller, G., 1978. On and off effects in the background EEG activity during one-second photic stimulation. Electroenceph. Clin. Neurophysiol. 44, 307-316

Babiloni, C., Vecchio, F., Miriello, M., Romani, G.L., Rossini, P.M., 2006. Visuo-spatial consciousness and parieto-occipital areas: a high-resolution EEG study. Cereb. Cortex 16, 37-46. 10.

Baker, J.T., Donoghue, J.P., Sanes, J.N., 1999. Gaze direction modulates finger movement activation patterns in human cerebral cortex. J. Neurosci. 19 (22), 10044-10052.

Barnikol, U.B., Amunts, K., Dammers, J., Mohlberg, H., Fieseler, T., Malikovic, A., Zilles, K., Niedeggen, M., Tass, P.A., 2006. Pattern reversal visual evoked re- 
sponses of V1/V2 and V5/MT as revealed by MEG combined with probabilistic cytoarchitectonic maps. Neuroimage 31 (1), 86-108.

Baselli, G., Porta, A., Rimoldi, O., Pagani, M., Cerutti, S., 1997. Spectral decomposition in multichannel recordings based on multivariate parametric identification. IEEE Trans. Biomed. Eng. 44, 1092-1101.

Binkofski, F., Buccino, G., 2006. The role of ventral premotor cortex in action execution and action understanding. J. Physiol. Paris. 99, 396-405.

Binkofski, F., Dohle, C., Posse, S., Stephan, K.M., Hefter, H., Seitz, R.J., Freund, H.-J., 1998. Human anterior intraparietal area subserves prehension. A combined lesion and functional MRI activation study. Neurology 50, 1253-1259.

Boussaoud, D., Wise, S.P., 1993. Primate frontal cortex: neuronal activity following attentional versus intentional cues. Exp. Brain Res. 95, 15-27.

Boussaoud, D., 2001. Attention versus intention in the primate premotor cortex. Neuroimage $14, \mathrm{~S} 40-\mathrm{S} 45$

Bradley, W.G., Daroff, R.B., Fenichel, G.M., Marsden, C.D., 2000. Neurology in Clinical Practice, 1. Butterworth-Heinemann, Oxford.

Brovelli, A., Ding, M., Ledberg, A., Chen, Y., Nakamura, R., Bressler, S.L., 2004. Beta oscillations in a large-scale sensorimotor cortical network: directional influences revealed by Granger causality. Proc. Natl. Acad. Sci. USA 101, 9849-9854.

Cadoret, G., Smith, A.M., 1997. Comparison of the neuronal activity in the SMA and the ventral cingulate cortex during prehension in the monkey. J. Neurophysiol. 77 (1), 153-166.

Cadoret, G., Smith, A.M., 1995. Input- output properties of hand-related cells in the ventral cingulate cortex in the monkey. J. Neurophysiol. 73, 2584-2590.

Castiello, U., 2005. The Neuroscience of Grasping. Nat. Rev. Neurosci. 6, 726-736.

Castiello, U., Begliomini, C., 2008. The cortical control of visually guided grasping. Neuroscientist 14, 157-170.

Chen, W.H., Mima, T., Siebner, H.R., Oga, T., Hara, H., Satow, T., Begum, T., Nagamine, T., Shibasaki, H., 2003. Low-frequency rTMS over lateral premotor cortex induces lasting changes in regional activation and functional coupling of cortical motor areas. Clin. Neurophysiol. 114, 1628-1637.

Cheyne, D., Bakhtazad, L., Gaetz, W., 2006. Spatiotemporal mapping of cortical activ ity accompanying voluntary movements using an event-related beamforming approach. Hum. Brain Mapp. 27, 213-229.

Classen, J., Gerloff, C., Honda, M., Hallett, M., 1998. Integrative visuomotor behaviour is associated with interregionally coherent oscillations in the human brain. $\mathrm{J}$. Neurophysiol. 79, 1567-1573.

Courtemanche, R., Fujii, N., Graybiel, A.M., 2003. Synchronous, focally modulated beta-band oscillations characterize local field potential activity in the striatum of awake behaving monkeys. J. Neurosci. 23, 11741-11752.

Davare, M., Montague, K., Olivier, E., Rothwell, J.C., Lemon, R.N., 2009. Ventral premotor to primary motor cortical interactions during object-driven grasp in humans. Cortex 45, 1050-1057.

Ehrsson, H.H., Fagergren, A., Jonsson, T., Westling, G., Johansson, R.S., Forssberg, H., 2000. Cortical activity in precision- versus power-grip tasks: an fMRI study. J. Neurophysiol. 83, 528-536.

Eickhoff, S.B., Heim, S., Zilles, K., Amunts, K., 2006. Testing anatomically specified hypotheses in functional imaging using cytoarchitectonic maps. NeuroImage $32,570-582$.

Eickhoff, S.B., Stephan, K.E., Mohlberg, H., Grefkes, C., Fink, G.R., Amunts, K., Zilles, K., 2005. A new SPM toolbox for combining probabilistic cytoarchitectonic maps and functional imaging data. Neuroimage 25, 1325-1335

Faes, L., Pinna, G.D., Porta, A., Maestri, R., Nollo, G., 2004. Surrogate data analysis for assessing the significance of the coherence function. IEEE Trans. Biomed. Eng. 51, 1156-1166.

Fogassi, L., Gallese, V., Buccino, G., Craighero, L., Fadiga, L., Rizzolatti, G., 2001. Cortical mechanism for the visual guidance of hand grasping movements in the monkey: a reversible inactivation study. Brain 124, 571-586.

Flanagan, J.R., Burstedt, M.K., Johansson, R.S., 1999. Control of fingertip forces in multidigit manipulation. J. Neurophysiol. 81 (4), 1706-1717.

Floyer-Lea, A., Matthews, P.M., 2004. Distinguishable brain activation networks for short- and long-term motor skill learning. J. Neurophysiol. 94 (1), 512-518.

Formaggio, E., Storti, S.F., Avesani, M., Cerini, R., Milanese, F., Gasparini, A., Acler, M., Pozzi, Mucelli, R., Fiaschi, A., Manganotti, P., 2008. EEG and FMRI coregistration to investigate the cortical oscillatory activities during finger movement Brain Topogr. 21 (2), 100-111.

Gallese, V., Murata, A., Kaseda, M., Niki, N., Sakata, H., 1994. Deficit of hand preshaping after muscimol injection in monkey parietal cortex. Neuroreport $5,1525-1529$.

Garavan, H., Ross, T.J., Kaufman, J., Stein, E.A., 2003. A midline dissociation between error-processing and response-conflict monitoring. Neuroimage 20 (2), $1132-1139$

Gastaut, H., 1952. Etude electrocorticographique de la reactivite des rhythmes rolandiques. Rev. Neurol. 87, 176-182.

Goodale, M.A., 2008. Action without perception in human vision. Cogn. Neuropsychol. 25, 891-919.

Goodale, M.A., Milner, A.D., 1992. Separate visual pathways for perception and action. Trends Neurosci. 15, 20-25.
Grafton, S.T., Fagg, A.H., Arbib, M.A., 1998. Dorsal premotor cortex and conditiona movement selection: a PET functional mapping study. J. Neurophysiol. 79 (2), 1092-1097.

Gray, C.M., Konig, P., Engel, A.K., Singer, W., 1989. Oscillatory responses in cat visual cortex exhibit inter-columnar synchronization which reflects global stimulus properties. Nature 338, 334-337.

Harrison, L., Penny, W.D., Friston, K., 2003. Multivariate autoregressive modeling of fMRI time series. Neuroimage 19 (4), 1477-1491.

Hepp-Reymond, M.C., 1988. Functional organization of motor cortex and its participation in voluntary movements. Comp. Primate Biol. 4, 501-624.

Hester, R., Foxe, J.J., Molholm, S., Shpaner, M., Garavan, H., 2005. Neural mechanisms involved in error processing: a comparison of errors made with and without awareness. Neuroimage 27 (3), 602-608.

Huang, M.X., Mosher, J.C., Leahy, R.M., 1999. A sensor-weighted overlapping-sphere head model and exhaustive head model comparison for MEG. Phys. Med. Biol. 44, 423-440.

Iacoboni, M., Zaidel, E., 2004. Interhemispheric visuo-motor integration in humans: the role of the superior parietal cortex. Neuropsychologia 42 (4), 419-425.

Iacoboni, M., Lieberman, M.D., Knowlton, B.J., Molnar-Szakacs, I., Moritz, M. Throop, C.J., Fiske, A.P., 2004. Watching social interactions produces dorsomedial prefrontal and medial parietal BOLD fMRI signal increases compared to a resting baseline. Neuroimage 21 (3), 1167-1173.

Inoue, K., Kawashima, R., Satoh, K., Kinomura, S., Goto, R., Koyama, M., et al., 1998. PET study of pointing with visual feedback of moving hands. J. Neurophysiol. 79, 117-125.

Jantzen, K.J., Fuchs, A., Mayville, J.M., Deecke, L., Kelso, J.A., 2001. Neuromagnetic activity in alpha and beta bands reflect learning-induced increases in coordinative stability. Clin. Neurophysiol. 112 (9), 1685-1697.

Jeannerod, M., Paulignan, Y., Weiss, P., 1998. Grasping an object: one movement, several components. Novartis Found Symp. 218, 5-16. discussion 16-20.

Jensen, O., Mazaheri, A., 2010. Shaping functional architecture by oscillatory alpha activity: gating by inhibition. Front Hum. Neurosci. 4, 186

Jerbi, K., Lachaux, J.P., N'Diaye, K., Pantazis, D., Leahy, R.M., Garnero, L., Baillet, S., 2007. Coherent neural representation of hand speed in humans revealed by MEG imaging. Proc. Natl. Acad. Sci. USA 104 (18), 7676-7681.

Johansson, R.S., 1996. Sensory control of dexterous manipulation in humans. In: Wing, A., Haggard, P., Flanagan, J. (Eds.), Hand and Brain. Academic, San Diego, pp. 381-414.

Jouffrais, C., Boussaoud, D., 1999. Neuronal activity related to eye-hand coordination in the primate premotor cortex. Exp. Brain Res. 128 (1-2), 205-209.

Kay, S.M., 1988. In Modern Spectral Estimation. Prentice Hall, Englewood Cliffs, New Jersey.

Kawashima, R., Matsumura, M., Sadato, N., Naito, E., Waki, A., Nakamura, S., Matsunami, K., Fukuda, H., Yonekura, Y., 1998. Regional cerebral blood flow changes in human brain related to ipsilateral and contralateral complex hand movements—a PET study. Eur. J. Neurosci. 10, 2254-2260.

Kelso, J.A.S., Fuchs, A., Lancaster, R., Holroyd, T., Cheyne, D., Weinberg, H., 1998 Dynamic cortical activity in the human brain reveals motor equivalence. Nature 392, 814-818

Kiehl, K.A., Liddle, P.F., Hopfinger, J.B., 2000. Error processing and the rostral anterior cingulate: an event-related fMRI study. Psychophysiology 37 (2), 216-223.

Klostermann, F., Nikulin, V.V., Kühn, A.A., Marzinzik, F., Wahl, M., Pogosyan, A., Kupsch, A., Schneider, G.H., Brown, P., Curio, G., 2007. Task-related differential dynamics of EEG alpha- and beta-band synchronization in cortico-basal motor structures. Eur. J. Neurosci. 25 (5), 1604-1615.

Konishi, S., Chikazoe, J., Jimura, K., Asari, T., Miyashita, Y., 2005. Neural mechanism in anterior prefrontal cortex for inhibition of prolonged set interference. Proc. Natl. Acad. Sci. USA 102, 12584-12588.

Kotra, L.P., Xiang, Y., Newton, M.G., Schinazi, R.F., Cheng, Y.C., Chu, C.K., 1997 Structure-activity relationships of $2^{\prime}$-deoxy-2', $2^{\prime}$-difluoro-L-erythro-pentofuranosyl nucleosides. J. Med Chem. 40 (22), 3635-3644.

Kranczioch, C., Athanassiou, S., Shen, S., Gao, G., Sterr, A., 2008. Short-term learning of a visually guided power-grip task is associated with dynamic changes in EEG oscillatory activity. Clin. Neurophysiol. 119 (6), 1419-1430.

Kristeva-Feige, R., Feige, B., Makeig, S., Ross, B., Elbert, T., 1993. Oscillatory brain activity during a motor task. Neuroreport 4 (12), 1291-1294.

Kuypers, H.G.J.M., 1981. Anatomy of the descending pathways. Handbook of Physiology. The Nervous System I. American Physiological Society, Bethesda, 597-666.

Lebedev, M.A., Wise, S.P., 2002. Insights into seeing and grasping: distinguishing the neural correlates of perception and action. Behav. Cogn. Neurosci. Rev. 1, 108-129.

Ledberg, A., Bressler, S.L., Ding, M., Coppola, R., Nakamura, R., 2007. Large-scale visuomotor integration in the cerebral cortex. Cereb. Cortex 17 (1), 44-62.

Lee, D., 2003. Coherent oscillations in neuronal activity of the supplementary motor area during a visuomotor task. J. Neurosci. 23 (17), 6798-6809.

Li, T., Xu, K., Deng, H., Cai, G., Liu, J., Liu, X., Wang, R., Xiang, X., Zhao, J., Murray, R.M., Sham, P.C., Collier, D.A., 1997. Association analysis of the dopamine 
D4 gene exon III VNTR and heroin abuse in Chinese subjects. Mol. Psychiatry 2 (5), 413-416.

Liu, L., Ioannides, A.A., 2010. Emotion separation is completed early and it depends on visual field presentation. PLoS One 5 (3), e9790.

Machado, S., Cunha, M., Velasques, B., Minc, D., Teixeira, S., Domingues, C.A., Silva, J.G., Bastos, V.H., Budde, H., Cagy, M., Basile, L., Piedade, R., Ribeiro, P., 2010. Sensorimotor integration: basic concepts, abnormalities related to movement disorders and sensorimotor training-induced cortical reorganization. Rev. Neurol. 51, 427-436.

MacKay, W.A., Mendonca, A.J., 1995. Field potential oscillatory bursts in parietal cortex before and during reach. Brain Res 704, 167-174.

Maier, M.A., Bennett, K.M., Hepp-Reymond, M.C., Lemon, R.N., 1993. Contribution of the monkey corticomotoneuronal system to the control of force in precision grip. J. Neurophysiol. 69 (3), 772-785.

Makris, N., Kennedy, D.N., McInerney, S., Sorensen, A.G., Wang, R., Caviness, V.S., Jr, Pandya, D.N., 2005. Segmentation of subcomponents within the superior longitudinal fascicle in humans: a quantitative, in vivo, DT-MRI study. Cereb. Cortex $15,854-869$.

Makris, N., Papadimitriou, G.M., Sorg, S., Kennedy, D.N., Caviness, V.S., Pandya, D.N., 2007. The occipitofrontal fascicle in humans: a quantitative, in vivo, DT-MRI study. Neuroimage 37, 1100-1111.

Matelli, M., Luppino, G., Rizzolatti, G., 1985. Patterns of cytochrome oxidase activity in the frontal agranular cortex of the macaque monkey. Behav. Brain Res. 18, 125-136.

Matsumura, M., Chen, D., Sawaguchi, T., Kubota, K., Fetz, E.E., 1996. Synaptic interactions between primate precentral cortex neurons revealed by spike-triggered averaging of intracellular membrane potentials in vivo. J. Neurosci. 16, 7757-7767.

Mayville, J.M., Fuchs, A., Ding, M., Cheyne, D., Deecke, L., Kelso, J.A., 2001. Event-related changes in neuromagnetic activity associated with syncopation and synchronization timing tasks. Hum. Brain Mapp. 14 (2), 65-80.

Menon, V., Adleman, N.E., White, C.D., Glover, G.H., Reiss, A.L., 2001. Error-related brain activation during a Go/NoGo response inhibition task. Hum. Brain Mapp. 12 (3), 131-143.

Meiran, N., Chorev, Z., Sapir, A., 2000. Component processes in task switching. Cogn. Psychol. 41, 211-253.

Miller, E.K., Cohen, J.D., 2001. An integrative theory of prefrontal cortex function. Annu. Rev. Neurosci. 24, 167-202.

Milner, A.D., Goodale, M.A., 2008. Two visual systems re-viewed. Neuropsychologia 46, 774-785.

Murthy, V.N., Fetz, E.E., 1996. Synchronization of neurons during local field potential oscillations in sensorimotor cortex of awake monkeys. J. Neurophysiol. 76 (6), 3968-3982.

Mylonas, D.S., Siettos, C.I., Evdokimidis, I., Papanicolaou, A.C., Smyrnis, N., 2016. Modular Patterns of Phase Desynchronization Networks During a Simple Visuomotor Task. Brain Topogr. (In Press).

Neuper, et al., 2007. Imagery and motor actions: differential effects of kinesthetic and visual-motor mode of imagery in single-trial EEG. Cogn. Brain Res. 25 (3), 668-677.

Neuper, C., Pfurtscheller, G., 2001. Evidence for distinct beta resonance frequencies in human EEG related to specific sensorimotor cortical areas. Clin. Neurophysiol. 112 (11), 2084-2097.

Nichols, T.E., Holmes, A.P., 2002. Nonparametric permutation tests for functional neuroimaging: a primer with examples. Hum. Brain Mapp. 15 (1), 1-25

Ohara, S., Ikeda, A., Kunieda, T., Yazawa, S., Baba, K., Nagamine, T., et al., 2000. Movement-related change of electrocorticographic activity in human supplementary motor area proper. Brain 123 (6), 1203-1215.

Olivier, E., Davare, M., Andres, M., Fadiga, L., 2007. Precision grasping in humans: from motor control to cognition. Curr. Opin. Neurobiol. 17, 644-648.

Papadelis, C., Eickhoff, S.B., Zilles, K., Ioannides, A.A., 2011. BA3b and BA1 activate in a serial fashion after median nerve stimulation: direct evidence from combining source analysis of evoked fields and cytoarchitectonic probabilistic maps. Neuroimage 54, 60-73.

Papadelis, C., Poghosyan, V., Fenwick, P.B., Ioannides, A.A., 2009. MEG's ability to localise accurately weak transient neural sources. Clin. Neurophysiol. 120, 1958-1970.

Paradiso, G., Cunic, D., Saint-Cyr, J.A., Hoque, T., Lozano, A.M., Lang, A.E., 2004 Involvement of human thalamus in the preparation of self-paced movement. Brain 127, 2717-2731.

Pause, M., Kunesch, E., Binkofski, F., Freund, H.-J., 1989. Sensorimotor disturbances in patients with lesions of the parietal cortex. Brain 112, 1599-1625.

Petrides, M., Pandya, D.N., 2007. Efferent association pathways from the rostral prefrontal cortex in the macaque monkey. J. Neurosci. 27, 11573-11586.

Pfurtscheller, G., 1992. Event-related synchronization (ERS): an electrophysiological correlate of cortical areas at rest. Electro. Clin. Neurophysiol. 83 (1), 62-69.

Pfurtscheller, G., Aranibar, A., 1977. Event-related cortical desynchronization detected by power measurements of scalp EEG. Electro. Clin. Neurophysiol. 42 (6), $817-826$.

Pfurtscheller, G., Berghold, A., 1989. Patterns of cortical activation during planning of voluntary movement. Electro. Clin. Neurophysiol. 72 (3), 250-258.
Pfurtscheller, G., Leeb, R., Slater, M., 2006. Cardiac responses induced during thought-based control of a virtual environment. Int. J. Psychophysiol. 62, 134-14010.

Pfurtscheller, G., Lopes da Silva, F.H., 1999. Event-related EEG/MEG synchronization and desynchronization: basic principles. Clin. Neurophysiol. 110, 1842-1857.

Pfurtscheller, G., Neuper, C., 1997. Motor imagery activates primary sensorimotor area in humans. Neurosci. Lett. $239(2-3), 65-68$.

Pfurtscheller, G., Pregenzer, M., Neuper, C., 1994. Visualization of sensorimotor areas involved in preparation for hand movement based on classification of $\mathrm{mu}$ and central beta rhythms in single EEG trials in man. Neurosci. Lett. 181 (1-2), 43-46.

Picard, N., Smith, A.M., 1992. Primary motor cortical responses to perturbations of prehension in the monkey. J. Neurophysiol. 68 (5), 1882-1894.

Pistohl, T., Schulze-Bonhage, A., Aertsen, A., Mehring, C., Ball, T., 2012. Decoding natural grasp types from human ECoG. NeuroImage 59 (1), 248-260.

Polli, F.E., Barton, J.J., Cain, M.S., Thakkar, K.N., Rauch, S.L., Manoach, D.S., 2005. Rostral and dorsal anterior cingulate cortex make dissociable contributions during antisaccade error commission. Proc. Natl. Acad. Sci. USA 102 (43), 15700-15705.

Porter, R., Lemon, R.N., 1993. Corticospinal Function and Voluntary Movement. Oxford Univ. Press, Oxford, UK

Rabbitt, P.M.A., 1966. Errors and error correction in choice response tasks. J. Exp. Psychol. 71, 264-272.

Ramnani, N., Owen, A.M., 2004. Anterior prefrontal cortex: insights into function from anatomy and neuroimaging. Nat. Rev. Neurosci. 5, 184-194.

Rao, S.M., Binder, J.R., Bandettini, P.A., Hammeke, T.A., Yetkin, F.Z., Jesmanowicz, A., Lisk, L.M., Morris, G.L., Mueller, W.M., Estkowski, L.D., Wong, E.C., Haughton, V.M., Hyde, J.S., 1993. Functional magnetic resonance imaging of complex human movements. Neurology 43, 2311-2318.

Rearick, M.P., Johnston, J.A., Slobounov, S.M., 2001. Feedback-dependent modulation of isometric force control: an EEG study in visuomotor integration. Brain Res. Cogn. Brain Res. 12 (1), 117-130.

Rickert, J., Oliveira, S.C., Vaadia, E., Aertsen, A., Rotter, S., et al., 2005. Encoding of movement direction in different frequency ranges of motor cortical local field potentials. J. Neurosci. 25 (39), 8815-8824.

Rilk, A.J., Soekadar, S.R., Sauseng, P., Plewnia, C., 2011. Alpha coherence predicts accuracy during a visuomotor tracking task. Neuropsychologia 49 (13), 3704-3709.

Rizzolatti, G., Fadiga, L., Matelli, M., Bettinardi, V., Paulesu, E., Perani, D., Fazio, F., 1996. Localization of grasp representations in humans by PET: 1. Observation versus execution. Exp. Brain Res. 111, 246-252.

Rizzolatti, G., Luppino, G., 2001. The cortical motor system. Neuron 31, 889-901.

Rizzolatti, G., Luppino, G., Matelli, M., 1998. The organization of the cortical motor system: new concepts. Electro. Clin. Neurophysiol. 106, 283-296.

Robinson, S., Vrba, J., 1998. Recent advances in biomagnetism. In: Yoshimoto, T., Kotani, M., Kuriki, S., Karibe, H., Nakasato, N. (Eds.). Sendai: Tohoku University Press, pp. 302-305.

Roelfsema, P.R., Engel, A.K., Konig, P., Singer, W., 1997. Visuomotor integration is associated with zero time-lag synchronization among cortical areas. Nature $385,157-161$.

Roland, P.E., Zilles, K., 1996. Functions and structures of the motor cortices in humans. Curr. Opin. Neurobiol. 6, 773-781.

Rowe, J.B., Toni, I., Josephs, O., Frackowiak, R.S., Passingham, R.E., 2000. The prefrontal cortex: response selection or maintenance within working memory?. Science $288,1656-1660$.

Rubia, K., Smith, A.B., Brammer, M.J., Taylor, E., 2003. Right inferior prefrontal cortex mediates response inhibition while mesial prefrontal cortex is responsible for error detection. NeuroImage 20 (1), 351-358.

Salenius, S., Portin, K., Kajola, M., Salmelin, R., Hari, R., 1997. Cortical control of human motoneuron firing during isometric contraction. J. Neurophysiol. 77 (6), 3401-3405.

Salmelin, R., Hämäläinen, M., Kajola, M., Hari, R., 1995. Functional segregation of movement-related rhythmic activity in the human brain. NeuroImage 2 (4), 237-243.

Salmelin, R., Hari, R., 1994. Spatiotemporal characteristics of sensorimotor neuromagnetic rhythms related to thumb movement. Neuroscience $60,537-550$.

Sanes, J.N., Donoghue, J.P., 1993. Oscillations in local field potentials of the primate motor cortex during voluntary movement. Proc. Natl. Acad. Sci. USA 90 (10), $4470-4474$.

Sarvas, J., 1987. Basic mathematical and electromagnetic concepts of the biomagnetic inverse problem. Phys. Med. Biol. 32 (1), 11-22.

Schnitzler, A., Gross, J., 2005. Normal and pathological oscillatory communication in the brain. Nat. Rev. Neurosci. 6, 285-296.

Scheler, G., Fischer, M.J., Genow, A., Hummel, C., Rampp, S., Paulini, A., Hopfengärtner, R., Kaltenhäuser, M., Stefan, H., 2007. Spatial relationship of source localizations in patients with focal epilepsy: comparison of MEG and EEG with a three spherical shells and a boundary element volume conductor model. Hum. Brain Mapp. 28 (4), 315-322.

Schnitzler, A., Salenius, S., Salmelin, R., Jousmäki, V., Hari, R., 1997. Involvement of primary motor cortex in motor imagery: a neuromagnetic study. Neuroimage 6 (3), 201-208. 
Seitz, R.J., Canavan, A.G., Yaguez, L., Herzog, H., Tellmann, L., Knorr, U., Huang, Y., Homberg, V., 1997. Representations of graphomotor trajectories in the human parietal cortex: evidence for controlled processing and automatic performance. Eur. J. Neurosci. 9, 378-389.

Singer, W., 1993. Synchronization of cortical activity and its putative role in information processing and learning. Annu. Rev. Physiol. 55, 349-374.

Singer, W., 1999. Neurobiology. Striving for coherence. Nature 397, 391-393.

Singh, K.D., Barnes, G.R., Hillebrand, A., 2003. Group imaging of task-related changes in cortical synchronisation using nonparametric permutation testing. Neuroimage $19,1589-1601$.

Singh, K.D., Barnes, G.R., Hillebrand, A., Forde, E.M., Williams, A.L., 2002. Task-related changes in cortical synchronization are spatially coincident with the hemodynamic response. Neuroimage 16,103-114.

Spraker, M.B., Corcos, D.M., Vaillancourt, D.E., 2009. Cortical and subcortical mechanisms for precisely controlled force generation and force relaxation. Cereb. Cortex 19, 2640-2650.

Stancák Jr., A., Pfurtscheller, G., 1996. Event-related desynchronisation of central beta-rhythms during brisk and slow self-paced finger movements of dominant and nondominant hand. Brain Res. Cogn. Brain Res. 4 (3), 171-183.

Styliadis, C., Ioannides, A.A., Bamidis, P.D., Papadelis, C., 2014. Amygdala responses to Valence and its interaction by arousal revealed by MEG. Int J. Psychophysiol. 93 (1), 121-133.

Styliadis, C., Ioannides, A.A., Bamidis, P.D., Papadelis, C., 2015. Distinct cerebellar lobules process arousal, valence and their interaction in parallel following a temporal hierarchy. Neuroimage 110, 149-161.

Sugawara, K., Onishi, H., Yamashiro, K., Kirimoto, H., Tsubaki, A., Suzuki, M., Tamaki, H., Murakami, H., Kameyama, S., 2013. Activation of the human premotor cortex during motor preparation in visuomotor tasks. Brain Topogr. 26 (4), 581-590.

Taniguchi, M., Kato, A., Fujita, N., Hirata, M., Tanaka, H., et al., 2000. Movement related desynchronization of the cerebral cortex studied with spatially filtered magnetoencephalography. NeuroImage 12, 298-306.
Taylor, S.F., Stern, E.R., Gehring, W.J., 2007. Neural systems for error monitoring: recent findings and theoretical perspectives. Neuroscientist 13 (2), 160-172.

Tomassini, V., Jbabdi, S., Klein, J.C., Behrens, T.E., Pozzilli, C., Matthews, P.M., Rushworth, M.F., Johansen-Berg, H., 2007. Diffusion-weighted imaging tractography-based parcellation of the human lateral premotor cortex identifies dorsal and ventral subregions with anatomical and functional specializations. J. Neurosci. 27, 10259-10269.

Traub, R.D., Whittington, M.A., Stanford, I.M., Jefferys, J.G., 1996. A mechanism for generation of long-range synchronous fast oscillations in the cortex. Nature 383, 621-624.

Tunik, E., Frey, S.H., Grafton, S.T., 2005. Virtual lesions of the anterior intraparietal area disrupt goal-dependent on-line adjustments of grasp. Nat. Neurosci. 8, 505-511.

Von der Malsburg, C., 1999. The what and why of binding: the modeler's perspective. Neuron 24, 95-104.

Von Stein, A., Sarnthein, J., 2000. Different frequencies for different scales of cortical integration: from local gamma to long range alpha/theta synchronization. Int. J. Psychophysiol. 38, 301-313.

Vaillancourt, D.E., Mayka, M.A., Corcos, D.M., 2006. Intermittent visuomotor processing in the human cerebellum, parietal cortex, and premotor cortex. J. Neurophysiol. 95, 922-931.

Wise, S.P., 2008. Forward frontal fields: phylogeny and fundamental function. Trends Neurosci. 31, 599-608

Wise, S.P., di Pellegrino, G., Boussaoud, D., 1992. Primate premotor cortex: dissociation of visuomotor from sensory signals. J. Neurophysiol. 68, 969-972.

Zaepffel, M., Trachel, R., Kilavik, B.E., Brochier, T., 2013. Modulations of EEG beta power during planning and execution of grasping movements. PLoS One 8 (3), e60060 